\title{
VEGF-C-dependent stimulation of lymphatic function ameliorates experimental inflammatory bowel disease
}

\author{
Silvia D'Alessio, ${ }^{1}$ Carmen Correale, ${ }^{1}$ Carlotta Tacconi, ${ }^{1}$ Alessandro Gandelli, ${ }^{1}$ Giovanni Pietrogrande, ${ }^{2}$ Stefania Vetrano, ${ }^{1}$ \\ Marco Genua, ${ }^{1}$ Vincenzo Arena, ${ }^{3}$ Antonino Spinelli, ${ }^{1}$ Laurent Peyrin-Biroulet, ${ }^{4}$ Claudio Fiocchi, ${ }^{5,6}$ and Silvio Danese ${ }^{1}$ \\ IIBD Center, Department of Gastroenterology, Humanitas Clinical and Research Center, Rozzano, Italy. ${ }^{2}$ Center for Translational Cenomics and Bioinformatics, San Raffaele University and Scientific Institute, \\ Milan, Italy. ${ }^{3}$ Institute of Pathology, Catholic University of the Sacred Heart, Rome, Italy. ${ }^{4}$ Department of Hepato-Gastroenterology, University Hospital of Nancy, Nancy, France. ${ }^{5}$ Department of \\ Gastroenterology and Hepatology, Digestive Disease Institute, and ${ }^{6}$ Department of Pathobiology, Lerner Research Institute, Cleveland Clinic Foundation, Cleveland, Ohio, USA.
}

Crohn's disease (CD) and ulcerative colitis (UC) are chronic inflammatory bowel diseases (IBDs) of unknown etiology that are associated with an aberrant mucosal immune response. Neoangiogenesis and vascular injury are observed in IBD along with increased lymphangiogenesis. While the pathogenic role of angiogenesis in IBD is well characterized, it is not clear how or if increased lymphangiogenesis promotes disease. Here, we determined that enhancing lymphangiogenesis and lymphatic function reduces experimental IBD. Specifically, we demonstrated that adenoviral induction of prolymphangiogenic factor VEGF-C provides marked protection against the development of acute and chronic colitis in 2 different animal models. VEGF-C-dependent protection was observed in combination with increased inflammatory cell mobilization and bacterial antigen clearance from the inflamed colon to the draining lymph nodes. Moreover, we found that the VEGF-C/VEGFR3

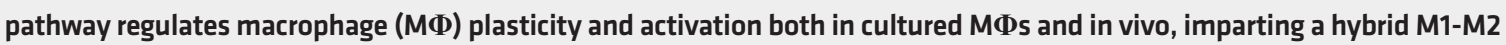
phenotype. The protective function of VECF-C was meditated by the so-called resolving MФs during chronic experimental colitis in a STAT6-dependent manner. Together, these findings shed light on the contribution of lymphatics to the pathogenesis of gut inflammation and suggest that correction of defective lymphatic function with VEGF-C has potential as a therapeutic strategy for IBD.

\section{Introduction}

The inflammatory bowel diseases (IBDs), consisting of ulcerative colitis (UC) and Crohn's disease (CD), are characterized by chronic inflammation of the gastrointestinal tract in genetically susceptible individuals exposed to environmental risk factors. The etiology of IBD has been extensively investigated in the past few decades, but its pathogenesis is not fully understood (1-3). While the study of IBD has been largely focused on the roles played by the classical components of the immune system, other reports have called attention to the significant contribution of nonimmune cells, such as epithelial, endothelial, mesenchymal, and vascular cells (4-6). In particular, there is a growing body of evidence showing that IBD is associated with the development of new blood (angiogenesis) and lymph (lymphangiogenesis) vessels from the existing vascular network (7-10). Although the pathogenic role of angiogenesis in IBD is well understood (11), whether lymphangiogenesis plays a protective or pathogenic role is still unknown. Recent studies demonstrated that lymphatic vessels (LVs) are functionally important for the resolution of intestinal inflammation. For example, mice deficient for angiopoietin-2 show intestinal lymphatic dysplasia and exacerbated colitis in the dextran sulfate sodium (DSS) colitis model $(12,13)$, while selective ablation of the LVs leads to distortion of the intestinal villi architecture and severe bowel inflammation

Conflict of interest: The authors have declared that no conflict of interest exists Submitted: July 17, 2013; Accepted: June 26, 2014.

Reference information: J Clin Invest. 2014;124(9):3863-3878. doi:10.1172/JCI72189.
(14). Although the precise pathophysiology of IBD is unknown, LV obstruction and dysfunction are long-recognized features observed in humans with this disease $(15,16)$. The existence of an abnormal lymphatic system is further supported by a recent article describing defective drainage capacity of LVs in human CD bowel strictures (17). Moreover, among patients with CD, relatively lower $\mathrm{LV}$ density was associated with a higher risk of endoscopic disease recurrence after surgery, suggesting that patients could benefit from improved lymphatic flow (18). In an animal model of intestinal ileitis, impaired contractile function of mesenteric collecting lymphatics was observed both in vivo and in vitro, again showing a strong correlation with the degree of mucosal inflammation (19). Nevertheless, together with LV dysfunction, macrophages (MФs) may also have a fundamental contribution to IBD pathogenesis. They have been found to play opposing roles in experimental models of intestinal inflammation, such as the DSS and IL-10-KO models of colitis $(20,21)$. In fact, while classically activated M1 MФs contribute to the pathogenesis of these 2 models of experimental colitis primarily by secreting proinflammatory cytokines, such as IL-6, TNF- $\alpha$ and IFN- $\gamma$, and causing tissue damage (22), alternatively activated M2 MФs contribute to the resolution of colitis primarily by expressing low levels of proinflammatory cytokines, but high levels of arginase 1 (Arg-1), FIZZ1, YM-1, and IL-10 (20, 23, 24). Thus, the factors that modulate $M \Phi$ polarization could affect the severity of human and experimental colitis. These observations suggest that resolution of chronic inflammation may require restoration of proper lymphatic function and proper $\mathrm{M} \Phi$ activation 
because of the essential importance of maintaining normal flow balance and removing inflammatory cells, mediators, and bacterial antigens away from inflamed sites (25).

Lymphangiogenesis is mediated by binding of the lymphatic vascular endothelial selective growth factors VEGF-C and VEGF-D to VEGFR3 $(26,27)$. VEGFR3, also known as Flt4, was the first lymphatic-specific growth factor receptor identified (28), and its neutralization by specific antibodies reduces bacterial infection associated-airway inflammation (29) and surgical blockade-induced lymphangiogenesis $(30,31)$. Moreover, antilymphatic treatment with anti-VEGFR3 antibodies in an animal model of IBD aggravates inflammation and submucosal edema, increases leukocyte infiltration, and causes the LVs to become tortuous $(13,32)$. VEGF-C is also chemotactic for MФs during pathological conditions, and its receptor VEGFR3 is expressed by a substantial fraction of peripheral blood monocytes and activated tissue MФs $(33,34)$. Overall, these studies have established a direct role for VEGF-C/VEGFR3 signaling in inflammation-induced lymphangiogenesis and immune response and suggest that therapies aimed at promoting lymphatic function, e.g., with prolymphangiogenic factors, such as VEGF-C, may provide a novel strategy for the treatment of inflammatory conditions, including IBD. In the current study, we examined the effect of stimulating lymphatic function and adaptive immune response via VEGF-C/VEGFR3 signaling on the severity of intestinal inflammation, on lymphatic drainage, as well as on bacterial antigen clearance and $\mathrm{M} \Phi$ activation during inflammatory conditions. Furthermore, we evaluated the mechanism through which this pathway acts in experimental disease progression.

\section{Results}

$L V$ density and the VEGF-C/VEGFR3 pathway are altered in active $I B D$-involved colons. To assess whether lymphangiogenesis occurs in human IBD, we performed immunohistochemical staining of control and active CD- and UC-involved colons with antibodies recognizing podoplanin, a widely accepted marker of lymphatic vasculature $(9,10,35)$. Normal colon mucosa only showed sporadic, thin LVs in the lamina propria and submucosa, whereas $C D$ and UC tissues contained numerous, readily detectable dilated LVs (Figure 1A). Quantitative analysis of the same sections revealed that the total number of LVs per field in the lamina propria and submucosa of both CD and UC specimens was significantly increased compared with that of the control non-IBD tissue (Figure 1B), thus confirming the occurrence of lymphangiogenesis in IBD patients.

The molecular mechanisms of inflammation-induced lymphangiogenesis remain largely unknown. Reports showing inhibition of LV growth by blockade of VEGFR3 signaling demonstrate the involvement of this receptor in inflammation-driven lymphangiogenesis (29). To verify whether the VEGF-C/VEGFR3 pathway is involved in intestinal inflammation-dependent lymphangiogenesis, control and active CD- and UC-involved colons were analyzed for the expression of these 2 molecules. Figure 1C (upper panel) shows that compared with control tissues, VEGF-C expression was upregulated in the mucosal extracts of patients with IBD. The higher VEGF-C levels observed in UC patients compared with those in $\mathrm{CD}$ patients may be the result of a greater degree of mucosal inflammation in the UC samples. Notably, the other VEGFR3 ligand VEGF-D showed comparable amounts in all groups (Figure 1C, lower panel), and for this reason, it was subsequently excluded from the study. Frozen sections double-stained with anti-LYVE1 and anti-VEGFR3 Abs showed increased VEGFR $^{+}$vessel density in inflamed tissue compared with that seen in quiescent LVs in control sections (Figure 1, D and E). Coexpression of VEGFR3 and LYVE1 was quantified using the method described by Tammela et al. (36), which measures the fluorescent intensity of target expression normalized per vascular area. This revealed a lymphatic-specific increase in VEGFR3 (Figure 1F), but not LYVE1 (Figure 1G), in CD and UC sections compared with that seen in control tissues, suggesting that inflammation also increases VEGFR3 expression in individual vessels. Collectively, these data demonstrate that mucosal lymphatic vascularization is increased in active IBD-involved tissues and suggest that chronic inflammation stimulates the VEGF-C/VEGFR3 pathway, a finding compatible with inflammation-associated lymphangiogenesis.

Role of VEGFR3 signaling in human intestinal lymphatic endothelial cell behavior in vitro. Proliferation, migration, and capillary reorganization are all processes necessary for lymphatic regeneration (37). To assess the role of VEGFR3 and its ligand VEGF-C in human intestinal lymphatic endothelial cell (HILEC) behavior, these cells were isolated from normal and IBD mucosa, as described in the Supplemental Information (supplemental material available online with this article; doi:10.1172/JCI72189DS1), and their biological functions were characterized in vitro. Medium supplemented with VEGF-C promoted the proliferation (Supplemental Figure 1A), migration (Supplemental Figure 1B), and capillary formation (Supplemental Figure 1, C and D) of both control (NL) and IBD HILECs, with a significantly greater response by IBD HILECs when compared with that by NL cells. VEGF156 mutant, a selective agonist that specifically binds VEGFR3, induced similar results, indicating that the effects of VEGF-C do not require VEGFR2 binding. After 96 hours of VEGF-C/VEGF156 treatment, blocking the activation of VEGFR3 (SAR131675) (38) significantly inhibited both basal and VEGF-C- or VEGF156-induced proliferation (Supplemental Figure 1A), migration (Supplemental Figure 1B), and tube formation (Supplemental Figure 1, C and D), with similar effects in NL and IBD HILECs, showing that VEGFR3 activation is necessary for these biological functions. We found that cell lysates from VEGF-C-stimulated NL and IBD HILECs displayed increased VEGFR3 phosphorylation (p-Tyr), which in turn was inhibited upon SAR131675 treatment (Supplemental Figure 1E). Moreover, overexpression of VEGFR3 in IBD HILECs confirmed what we observed by immunofluorescence (see Figure 1) and demonstrated that our method of isolation could represent a novel tool for in vitro study of lymphatics in IBD.

Systemic delivery of VEGF-C significantly improved experimental colitis both clinically and histologically. Since VEGFR3 signaling is essential for lymphangiogenesis in various conditions $(26,39)$, we sought to determine whether the pathway was also important for lymphangiogenesis in chronic experimental colitis. For this purpose, we studied the functional role of the VEGF-C/VEGFR3 pathway in vivo by systemic inhibition of VEGFR3 (15) or by delivery of the human lymphangiogenic factor VEGF-C in DSS and IL-10-KO models of chronic colitis using a blocking Ab or adenoviral transfer, respectively. Mice undergoing 2 cycles of DSS treatment and 15-week-old IL-10-KO animals with established colitis 
A
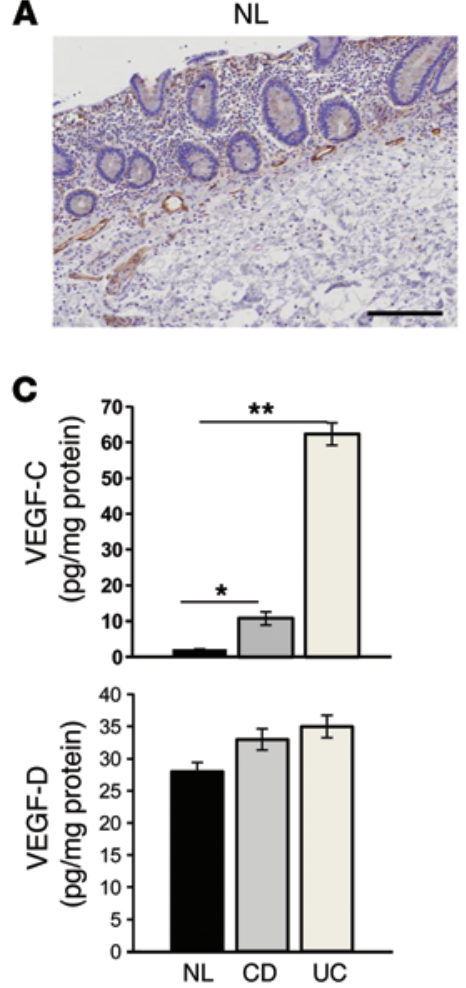

$C D$

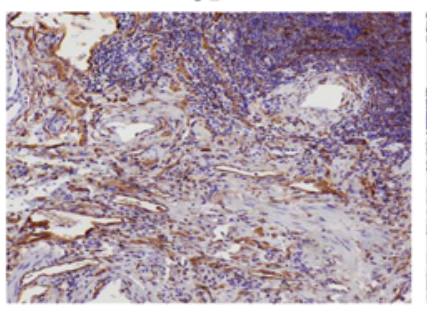

UC

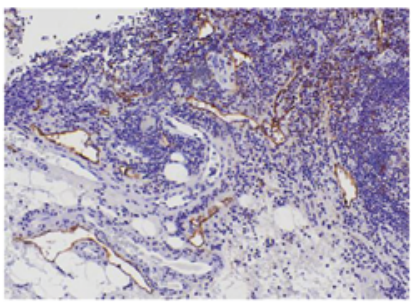

B

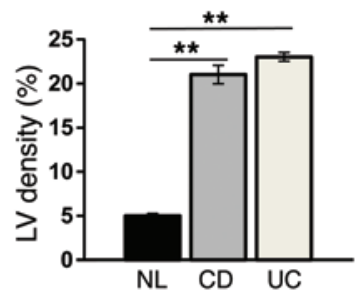

D

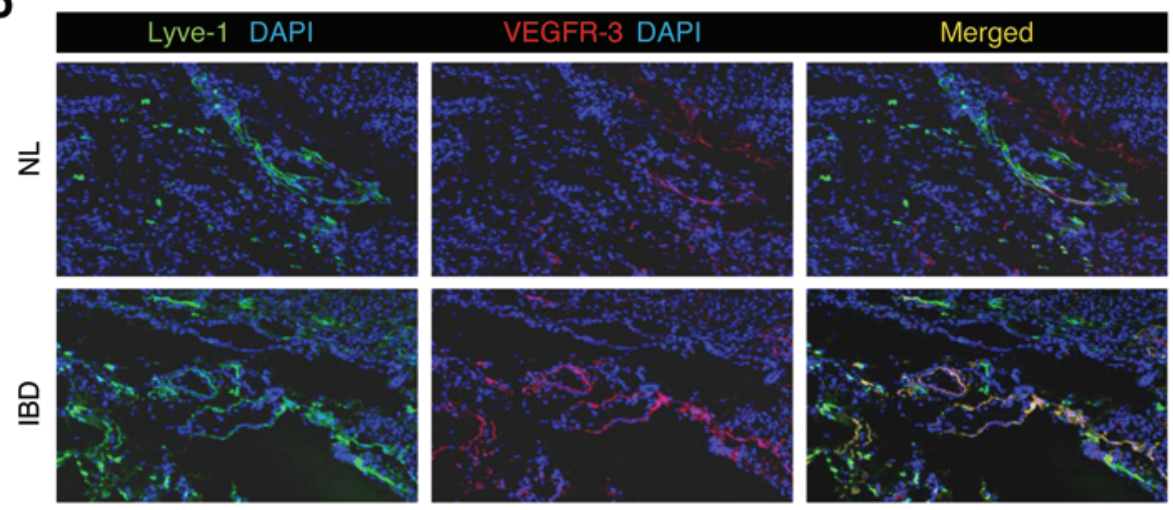

E

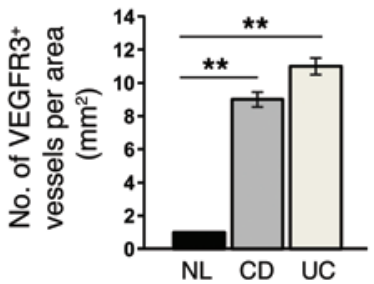

$\mathbf{F}$

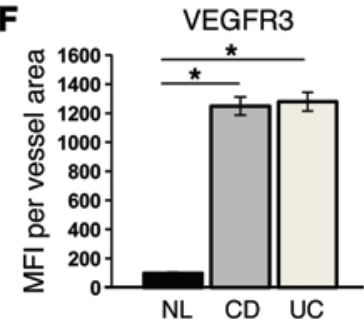

LYVE1

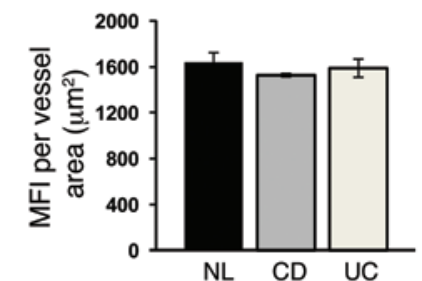

Figure 1. LV density and VEGF-C/VEGFR3 signaling are increased in the colon of IBD patients. (A and B) Immunohistochemical staining with an Ab recognizing podoplanin was performed on control $(\mathrm{NL})$ and active $\mathrm{CD}$ - and $\mathrm{UC}$-involved colons (A). Quantitative analysis of $\mathrm{LV}$ density in the same sections is shown in B. Data are expressed as the mean percentage of LV per area \pm SEM. Scale bar: $100 \mu \mathrm{m}$. (C) Control $(n=15)$ and CD $(n=16)$ and UC $(n=16)$ patients with active inflammation were investigated for VEGF-C and VEGF-D content in mucosal extracts. VEGF-C and VEGF-D were measured by ELISA. (D) Frozen sections from the colons of control $(\mathrm{NL}, n=15)$ or CD (IBD, $n=16)$ patients with active inflammation stained with both anti-LYVE1 and antiVEGFR3 Abs. Original magnification, $\times 40$. (E) VEGFR3 ${ }^{+}$LVs were quantified by stereological point counting of 10 regions per section, and the numbers were normalized per total section area expressed in $\mathrm{mm}^{2}$. Results are presented as the mean vessel density per group \pm SEM. (F and $\left.\mathbf{G}\right) \mathrm{MFI}$ per vessel was analyzed on VEGFR3 ${ }^{+}(\mathbf{F})$ and LYVE1 ${ }^{+}(\mathbf{C}) \mathrm{LVs}_{\mathrm{s}}(10-15$ vessels per section). MFI is expressed as relative units normalized per vascular area expressed in $\mu \mathrm{m}^{2}$. ${ }^{*} P<0.01$; ${ }^{* *} P<0.001$ (B, C, E, and F).

were injected with adenoviruses encoding human VEGF-C (AdhVEGF-C) or with the anti-VEGFR3 Ab mF431C1. Western blot and immunoprecipitation analyses of tissue lysates obtained from the colons of each experimental group clearly showed the efficient inhibition of VEGFR3 phosphorylation and upregulation of AdhVEGF-C at the indicated time points (Supplemental Figure 2A and data not shown). Notably, in the DSS model, we found that VEGFR3 protein levels were increased during both the acute and chronic phases of intestinal inflammation (Supplemental Figure 2A).

Systemic delivery of Ad-hVEGF-C significantly reduced the severity of chronic intestinal inflammation in both experimental models in terms of percentage of body weight (Figure 2, A and B), endoscopic evaluation (Figure 2C and Supplemental Figure 2B), and disease activity index (DAI, a combinatorial score including stool blood, form, and body weight) (Supplemental Figure 2, C and $\mathrm{D}$ ), whereas $\mathrm{mF} 431 \mathrm{C} 1$ worsened the clinical course compared with that of control groups (only DSS-treated and IL-10 WT mice). Notably, DSS-treated mice showed similar effects also in the acute phase of inflammation (Figure 2, A and E, and Supplemental Figure 2, B and D). Protection against chronic colitis by VEGF-C in these 2 experimental models was paralleled by histological examination, in which we also observed reduced inflammatory cell infiltration into the intestinal mucosa and diminished submucosal edema (Figure 2, D and E).

The VEGF-C/VEGFR3 pathway is required for lymphangiogenesis in experimental colitis. Lymphatics expanded dramatically during DSS-induced acute and chronic colitis. Frozen sections from the colon of control (untreated) or DSS-treated (colitic) mice were double stained with anti-LYVE1 and anti-VEGFR3 Abs, and VEGFR3 ${ }^{+}$ LVs were quantified in 10 regions per section. The results showed an increase in VEGFR3 ${ }^{+}$vessel density in inflamed tissues compared with LVs in control tissues (Supplemental Figure 3, A and B). 

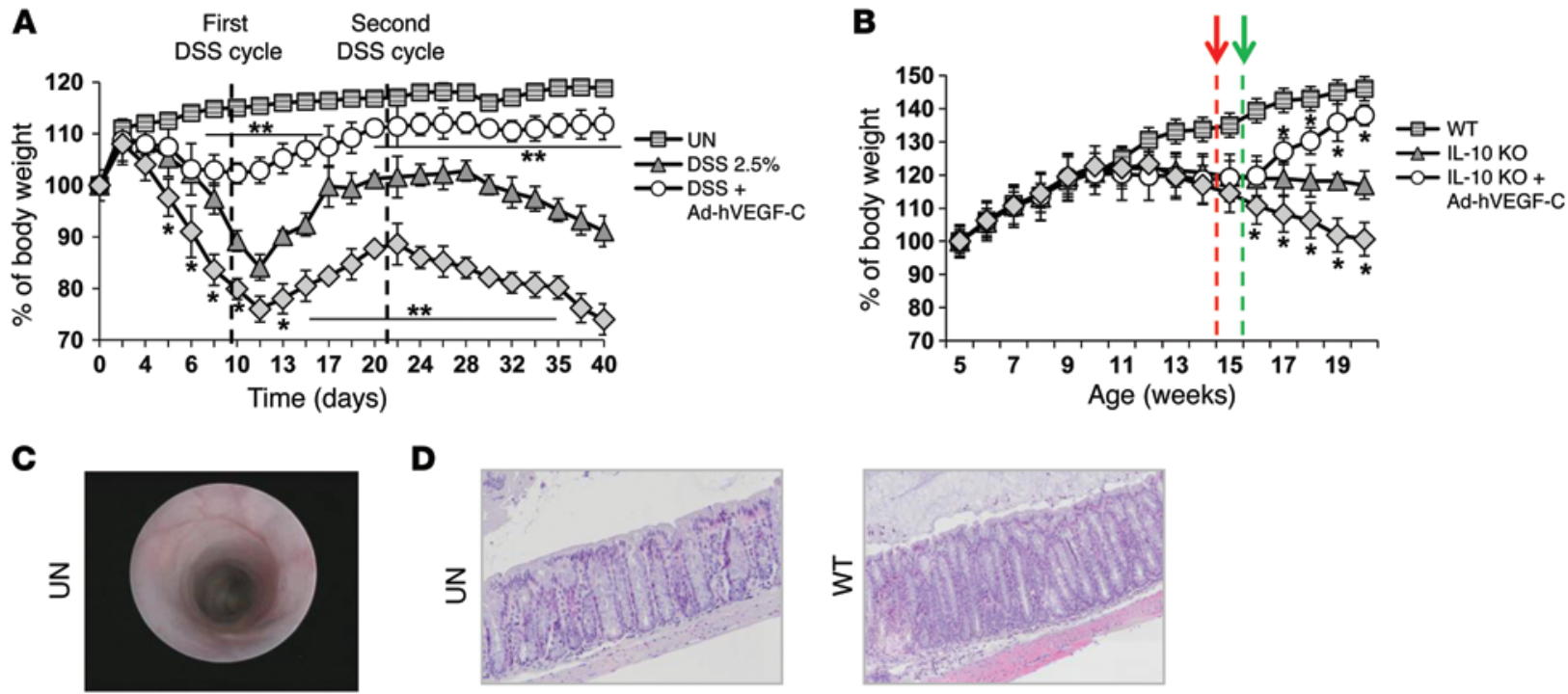

D
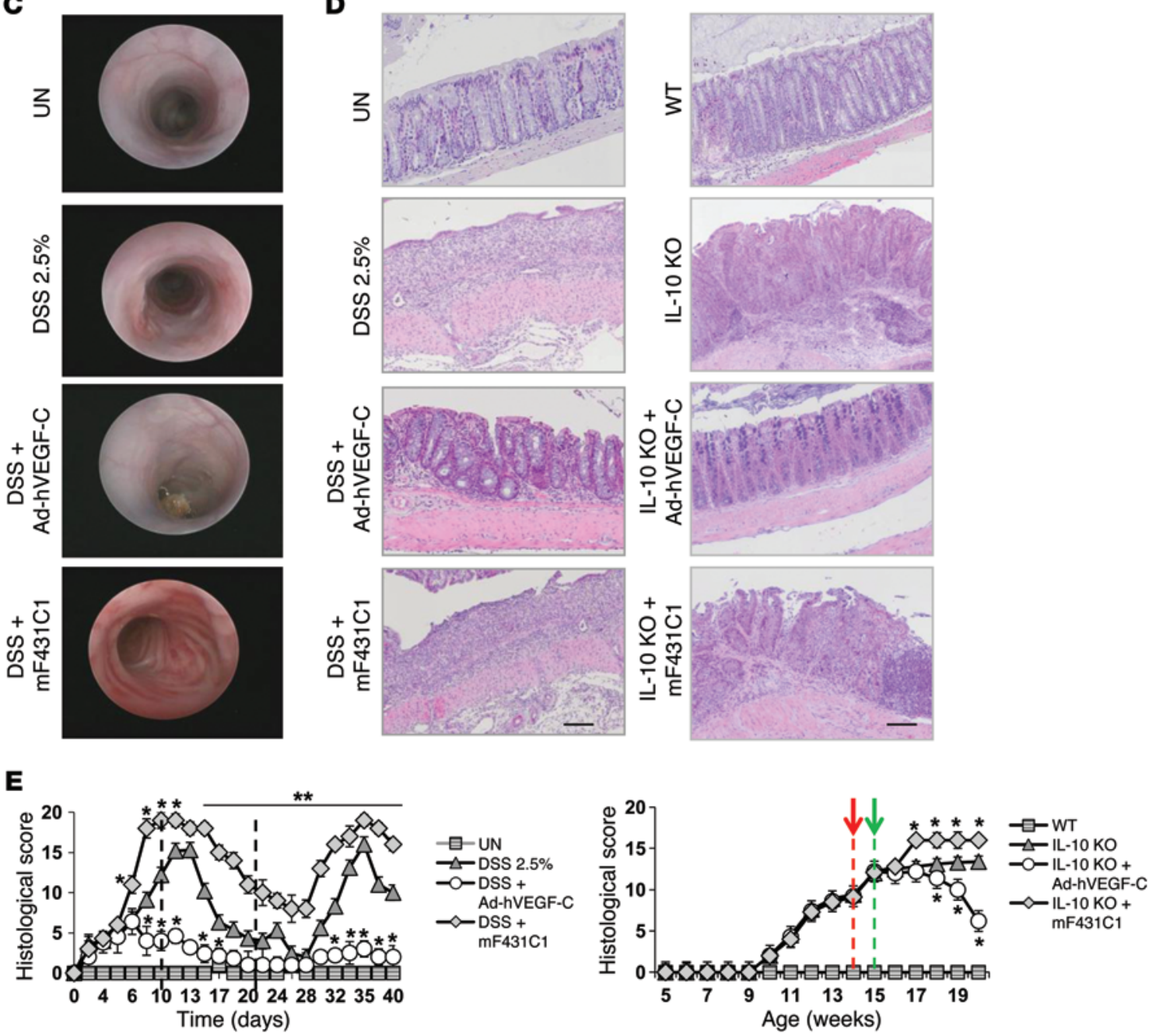

Figure 2. Systemic delivery of human VEGF-C significantly improves colitis both clinically and histologically. Mice undergoing 2 cycles of DSS treatment and 15-week-old IL-10-KO mice with established colitis were injected with Ad-hVEGF-C ( $n=8 /$ time point) or anti-VEGFR3 Ab (mF431C1, $n=8 /$ time point). Systemic delivery of VEGF-C significantly reduced the severity of colitis based on percentage of body weight (A and B), endoscopic evaluation (C), and histological examination ( $\mathbf{D}$ and $\mathbf{E}$ ), whereas mF431C1 worsened the colitis compared with that seen in control mice, which received DSS alone. UN, no DSS; WT, IL-10 WT. Black dashed lines represent the 2 DSS cycles. Red and green dashed lines indicate the first VEGF-C and mF431C1 administration, respectively. (C) Representative endoscopic images of colons from the indicated groups of mice after the second cycle of DSS (day 28). (D) Representative H\&Estained images of colons from the indicated groups of mice at the end of the experiment (day 40 for DSS-treated mice and week 20 for IL-10-KO animals). (E) Histological scores of the entire section were quantified blindly by an expert pathologist and were expressed as the mean value per group \pm SEM. All other graphs represent the mean value \pm SEM. ${ }^{*} P<0.05$ versus DSS alone; ${ }^{* *} P<0.001$ versus DSS alone.

Coexpression of VEGFR3 and LYVE1 was quantified, and the fluorescent intensity of target expression was normalized per vascular area. This revealed a lymphatic-specific increase in VEGFR3 (Supplemental Figure 3C) in colitic sections compared with that observed in control tissues, suggesting that inflammation also increases VEGFR3 levels in individual vessels. In parallel, protein extracts from the colon of colitic mice showed increased VEGF-C expression levels compared with those in healthy animals (Supple- 
A

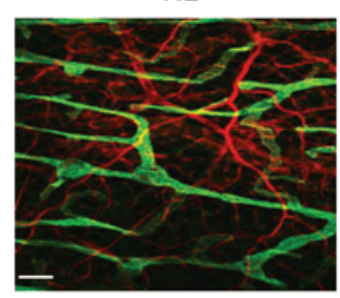

DSS $2.5 \%$

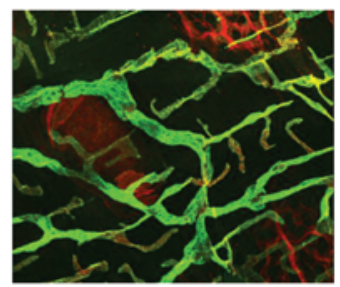

DSS $2.5 \%$ + Ad-hVEGF-C

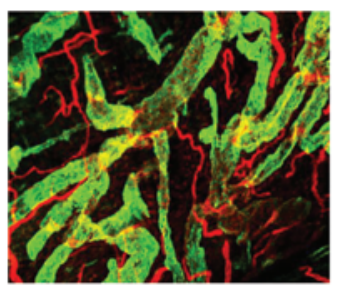

DSS $2.5 \%+m F 431 \mathrm{C} 1$

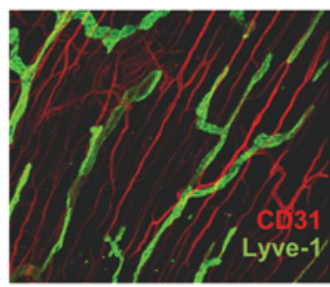

B

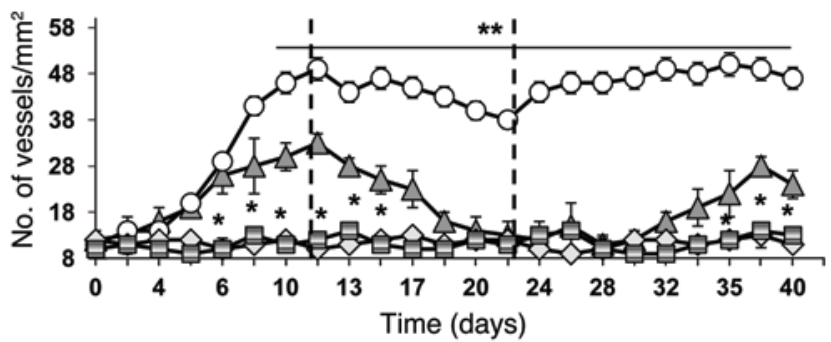

- DSS $2.5 \%$ $\rightarrow-$ DSS + Ad-hVEGF-C $\approx$ DSS + mF431C1 $-\mathrm{NL}$

C

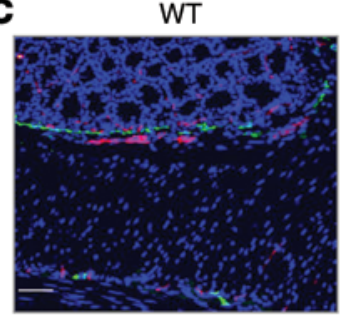

IL-10 KO

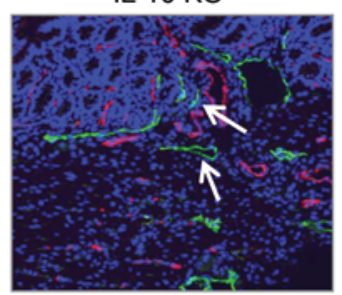

IL-10 KO + Ad-hVEGF-C

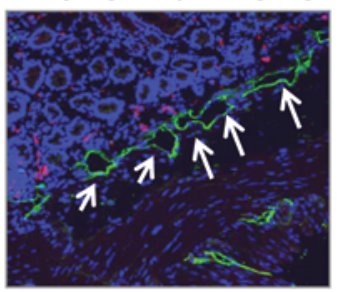

$\mathrm{IL}-10 \mathrm{KO}+\mathrm{mF} 431 \mathrm{C} 1$

D

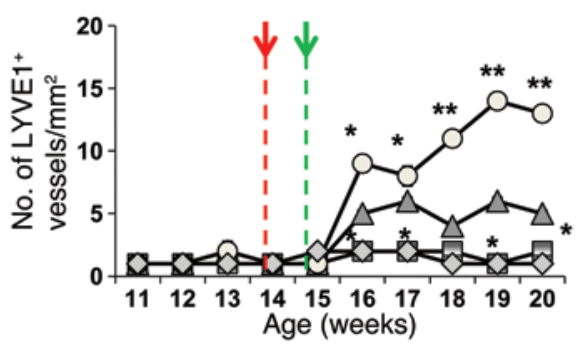

E

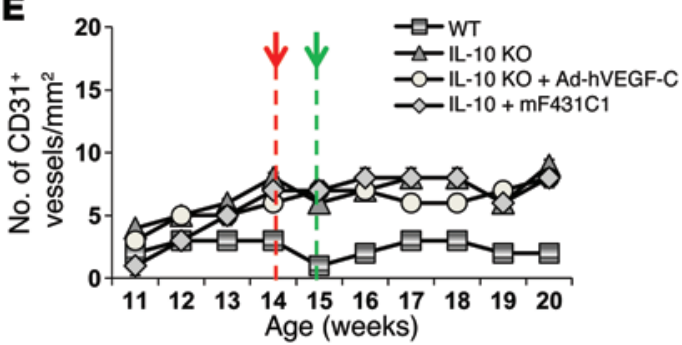

Figure 3. Systemic delivery of Ad-hVEGF-C increases LV density in colitic mice. Whole mounts of proximal, distal, and rectal colonic segments from DSS-treated mice with acute and chronic colitis were stained with Abs against LYVE1 (green) and CD31 (red) to measure LV area density. (A) Representative images of whole-mount staining of distal colons from the indicated groups at day 10. Scale bar: $75 \mu \mathrm{m}$. (B) LV density was quantified at the indicated time points and reported as the mean values \pm SEM for each group ( $n=8$ mice/group with 5 fields/colonic segment). Scale bar: $40 \mu \mathrm{m}$. ${ }^{*} P<0.05 ;{ }^{* *} P<0.001$ versus DSS alone. (C) Representative images of frozen sections from colons of treated control (WT, $n=8$ ) and IL-10-KO (colitic, $n=8$ ) mice stained for LYVE1 (green), CD31 (red), and DAPI (blue). Scale bar: $75 \mu \mathrm{m}$. LYVE1+(D) and CD31+ (E) vessels were counted in 10 regions per section, and the numbers were normalized per total section area expressed in $\mathrm{mm}^{2}$. Results are presented as the mean vessel density per group \pm SEM. Black dashed lines represent the 2 DSS cycles. Red and green dashed lines indicate the first VECF-C and mF431C1 administration, respectively. ${ }^{*} P<0.05$; ${ }^{*} P<0.001$ versus DSS alone.

mental Figure 3D), as observed in human tissues (see Figure 1). We obtained Similar results with IL-10-KO animals (data not shown).

Whole mounts of proximal, distal, and rectal colonic segments from DSS-treated and untreated mice were stained with Abs against LYVE1 and CD31 to measure the area density and dimension of LVs during acute and chronic colitis. We found that systemic delivery of VEGF-C significantly increased lymphangiogenesis at the indicated time points, both in terms of LV density (Figure 3, A and B) and size (Supplemental Figure 3E) in comparison with the DSS-only control group. In contrast, $\mathrm{mF} 431 \mathrm{C} 1 \mathrm{had}$ an inhibitory effect on LV dimension (Supplemental Figure 3E) and the number of LVs per area (Figure 3B). Frozen sections from colons of WT and colitic IL-10-KO mice were also double stained with anti-LYVE1 and anti-CD31 Abs (Figure 3C), and LYVE1 ${ }^{+}$LVs were counted in 10 regions per section at the indicated time points. Our results showed that while systemic delivery of VEGF-C significantly increased LV density, $\mathrm{mF} 431 \mathrm{C} 1$ reduced the number of LVs per area compared with that in the WT control group (Figure 3D). Notably, neither systemic inhibition of VEGFR3 nor delivery of the human lymphangiogenic factor VEGF-C affected the number of $\mathrm{CD} 31^{+}$blood vessels in the inflamed colon (Figure $3 \mathrm{E}$ and data not shown). This indicates that this pathway does not influence angiogenesis during experimental colitis. Besides, except for increased lymphangiogenesis in various organs, such as the 
A

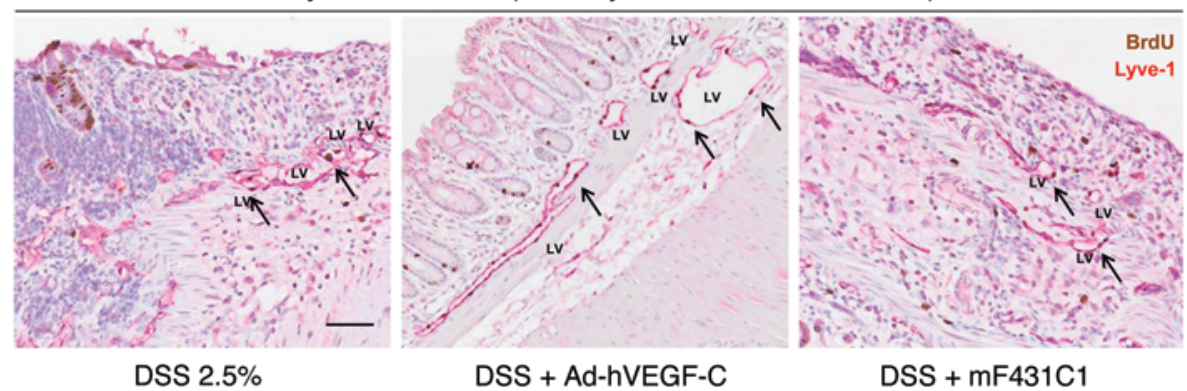

B

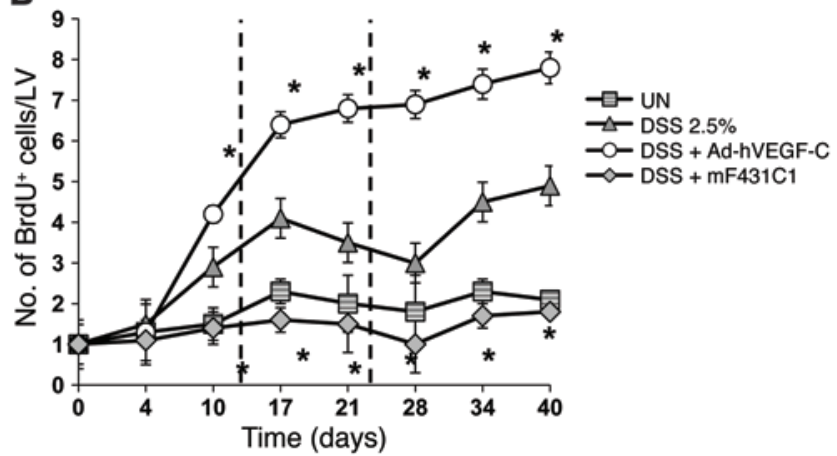

C

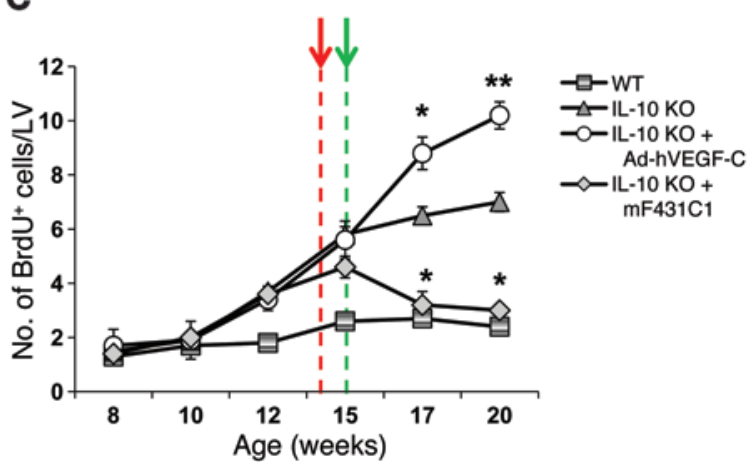

Figure 4. LV proliferation is increased in VEGF-C-treated colitic mice and blocked upon mF431C1 administration. Untreated ( $n=8 /$ time point) and VEGF-C- ( $n=8 /$ time point) or mF431C1-treated $(n=8)$ colitic mice were injected with BrdU 2 hours prior to sacrifice at the indicated time points. (A) Representative images of paraffin-embedded inflamed sections from DSS-treated mice were costained for BrdU (brown) in combination with anti-LYVE1 $\mathrm{Ab}(\mathrm{red})$, and the number of BrdU+ cells per LV was quantified in each group (B and C). Counts were performed on consecutive and randomly selected fields ( $n=10$ fields/section for each group), and data are reported as the mean \pm SEM. Black dashed lines represent the 2 DSS cycles. Red and green dashed lines indicate the first VEGF-C and mF431C1 administration, respectively. Scale bar: $28 \mu \mathrm{m} .{ }^{*} P<0.05 ;{ }^{* *} P<0.001$ versus DSS alone.

liver and spleen, we failed to find any evidence of inflammation or organ failure (data not shown), thus allowing us to conclude that no major systemic effects are expected upon treatment with this prolymphangiogenic factor. To determine the proliferative status of LVs at different stages of intestinal inflammation, untreated and VEGF-C- or mF431C1-treated colitic mice were injected with BrdU 2 hours prior to sacrifice. We costained the control and inflamed sections for BrdU in combination with anti-LYVE1 Ab and quantified the number of BrdU ${ }^{+}$cells per LV (Figure $4 \mathrm{~A}$ ). The results showed that systemic delivery of Ad-hVEGF-C enhanced LV proliferation in both the acute and chronic phases of DSSinduced colitis (Figure 4B) and in colitic IL-10-KO animals (Figure 4C) when compared with control groups (UN and WT, respectively). In contrast, $\mathrm{mF} 431 \mathrm{C} 1$ treatment completely inhibited LV growth in both experimental models (Figure 4, B and C). LV modulation correlated with disease activity and weight loss, demonstrating that the VEGF-C/VEGFR3 pathway is not only required for proper lymphangiogenesis during intestinal inflammation, but that lymphangiogenesis per se might be important for the resolution of inflammation.

Exacerbation of experimental colitis by VEGFR3 blockade is associated with decreased afferent lymph flow and inflammatory cell migration to draining nodes. Studies reporting abnormalities, such as submucosal edema, extensive dilation of lacteals $(40,41)$, lymphangitis, immune cell trafficking, and lymphangiogenesis $(9,42)$ in IBD patients suggest impaired lymph drainage and lym- phatic pumping, implicating the lymphatic system as a player in various inflammatory disorders, including IBD. For this reason, we next investigated whether inhibition of lymphangiogenesis in $\mathrm{mF} 431 \mathrm{C} 1$-treated mice is related to reduced lymphatic drainage and decreased inflammatory cell mobilization from the inflamed tissue to the draining lymph nodes (DLNs). To this end, we injected $10 \mu \mathrm{g}$ of Evans blue dye into the colonic mucosa of Ad-hVEGF-Ctreated and mF431C1-treated mice undergoing 2 cycles of DSS treatment, using DSS only-treated mice as a control group. Evans blue dye, which is specifically taken up by the lymphatic vasculature (43), was then extracted 16 hours after its injection. The extraction of Evans blue dye from distal colons of comparable weight revealed that although lymphatic drainage was decreased in the chronic phase of colitis when compared with healthy (untreated) mice, it was enhanced by systemic delivery of VEGF-C (Figure 5A). By contrast, mF431C1-treated mice had significantly more dye remaining in the inflamed tissue than did mice treated with DSS alone (Figure 5A), suggestive of reduced lymphatic clearance function. We obtained similar results with Ad-hVEGF-C- and mF431C1-treated IL-10-KO mice, using WT animals as a control group (Supplemental Figure 3F). We further determined the rate of inflammatory cell mobilization from healthy and inflamed colons to the DLNs by intramucosal injection of $\mathrm{GFP}^{+}$inflammatory cells (Figure 5B). Quantification of $\mathrm{GFP}^{+}$cells in the DLNs by immunofluorescence (Figure 5C, upper panels) or FACS analysis (Figure $5 \mathrm{C}$, lower panels) showed that systemic delivery of VEGF-C 
A

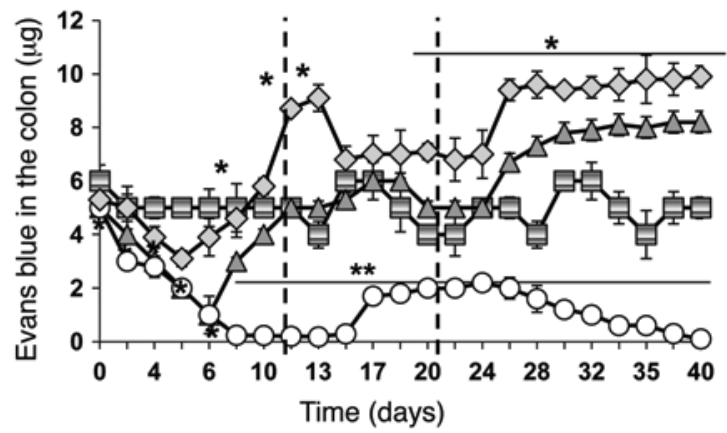

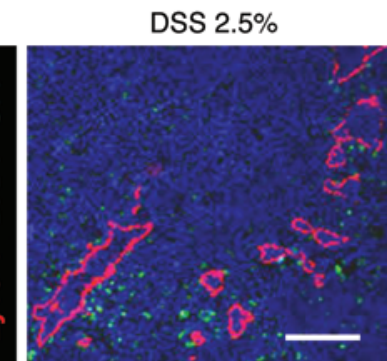

IL-10 KO

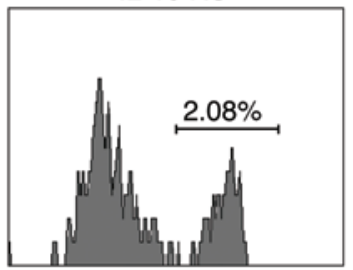

DSS + Ad-hVEGF-C

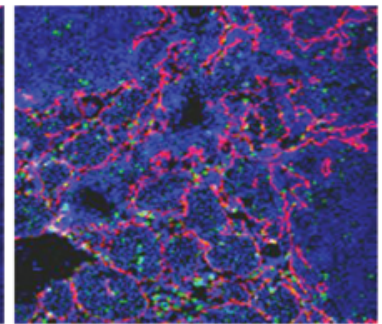

IL-10 KO + Ad-hVEGF-C

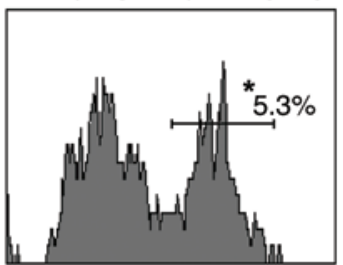

B

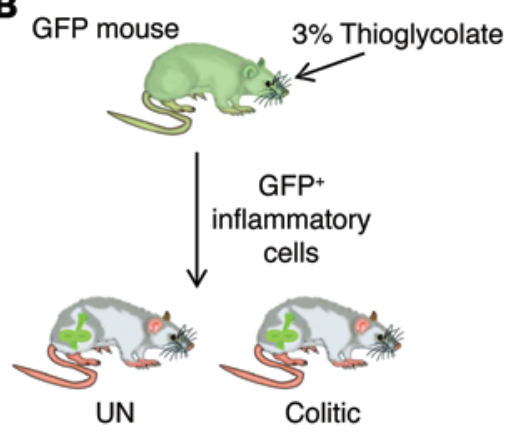

D

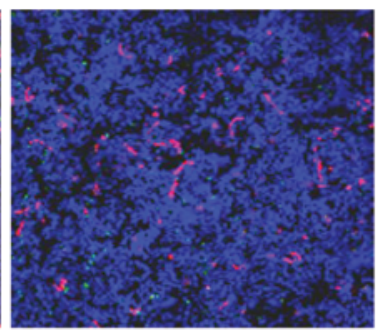

$\mathrm{IL}-10 \mathrm{KO}+\mathrm{mF} 431 \mathrm{C} 1$

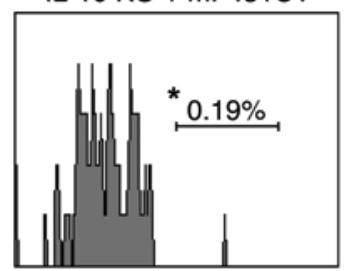

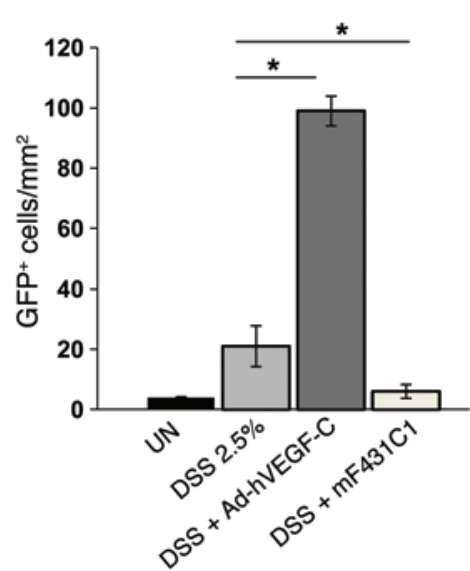

Figure 5. Systemic delivery of VEGF-C markedly increases lymphatic drainage and mobilization of inflammatory cells from the inflamed colon to DLNs. (A) $10 \mu \mathrm{g}$ of Evans blue dye was injected into the colonic mucosa of healthy (UN, $n=4 /$ time point), Ad-hVEGF-C- $(n=5 /$ time point), and mF431C1-treated mice $(n=5 /$ time point) undergoing 2 cycles of DSS treatment. Mice treated with DSS alone ( $n=5 /$ time point) were used as a control group. Evans blue was then extracted 16 hours after the dye injection from distal colons of comparable weight. Graph shows the total dye remaining in the colon expressed in $\mu$ g. Data represent the mean per group \pm SEM. Black dashed lines represent the $2 \mathrm{DSS}$ cycles. (B-D) GFP ${ }^{+}$peritoneal inflammatory cells $\left(\sim 10^{6}\right.$ cells) were injected into the rectal mucosa of the inflamed and noninflamed colons of healthy $(U N, n=5)$ and DSS-treated mice $(n=5$ per experimental group, at day 5 after the second DSS cycle) and IL-10 WT $(n=4)$ and IL-10-KO mice ( $n=4$ per group at day 21 after the first Ad-hVEGF-C administration). Twelve hours later, the DLNs were sampled and sectioned for histologic and FACS analysis. (C) Upper panels: Representative frozen sections of DLNs from the indicated experimental groups coimmunostained for LYVE1 (red) and DAPI (blue) and merged. Lower panels: Representative FACS from the indicated groups. The number in each FACS histogram represents the percentage of GFP+ cells. Scale bar: $50 \mu \mathrm{m}$. (D) GFP+ inflammatory cells in the entire frozen section were quantified and are presented as AU. Error bars represent the mean per group \pm SEM. ${ }^{*} P<0.05 ;{ }^{* *} P<0.001$.

considerably increased inflammatory cell migration, whereas systemic inhibition of the receptor VEGFR3 reduced the mobilization rate of inflammatory cells from the inflamed colon (Figure $5, \mathrm{C}$ and D). Overall, these data suggest that during experimental chronic colitis, the VEGF-C/VEGFR3 pathway plays a fundamental role in increasing lymph flow and inflammatory cell mobilization from the inflamed site to the DLNs, both of which are important events for inflammatory resolution.

Systemic delivery of VEGF-C accelerates antigen clearance from the inflamed colon to the DLNs through macrophage mobilization. One of the etiological theories on the pathogenesis of IBD is the impaired clearance of foreign material, leading to a sustained activation of innate immune cells and a compensatory induction of the adaptive immune response $(44,45)$. Indeed, delayed or ineffective clearance of bacteria that penetrate the intestinal mucosal barrier could prolong innate immune responses and enhance the presentation of bacterial antigens to facilitate the development of adaptive immune responses. Thus, while IBD may be due to an aberrant immune response against intestinal bacteria, slow or ineffective eradication of local bacteria may also be a key associated event contributing to disease pathogenesis (46). To ascertain whether the VEGF-C/ VEGFR3 pathway is involved in antigen clearance through local LVs and DLNs during intestinal inflammation, we administered a single intramucosal injection of carboxylated crimson fluorescent LPS-coated beads into colitic mice untreated or treated with AdhVEGF-C and mF431C1 and monitored the clearance of the LPScoated beads into the DLNs 1 and 3 days after injection. Two different populations of MФs and DCs were characterized based on the expression of CD11b, CD11c, F4/80, and CD103, and, to avoid overestimation due to cells that had taken up more beads, we selected only cells that had taken up 1 bead over all bead ${ }^{+}$cells (Figure 6A). While systemic delivery of VEGF-C accelerated the clearance of single-bead ${ }^{+}$cells from the inflamed colon (Figure 6, B and C, left panels) to the DLNs (Figure 6, B and C, right panels) both 1 and 
A

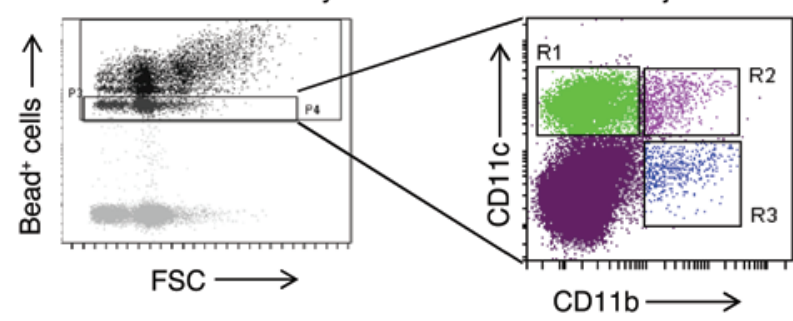

DLNs 3 days after LPS-coated bead injection
B

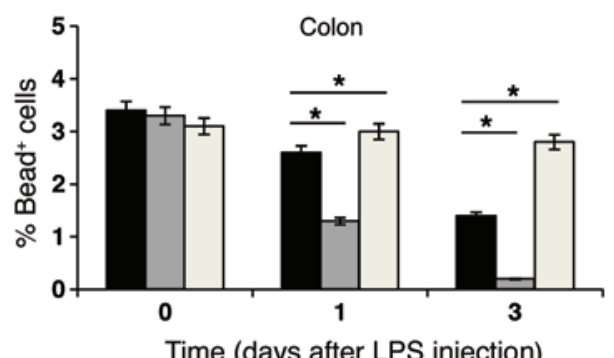

DSS

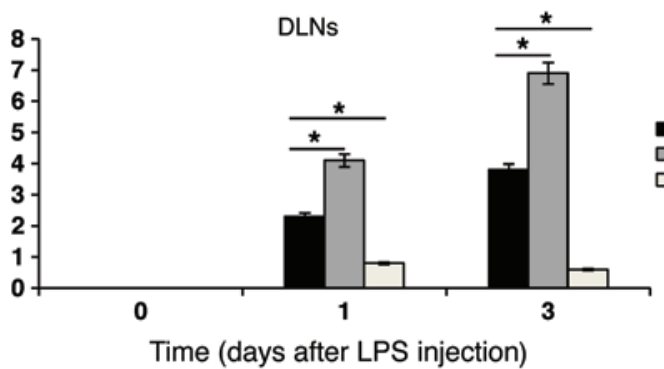

Gated on R2

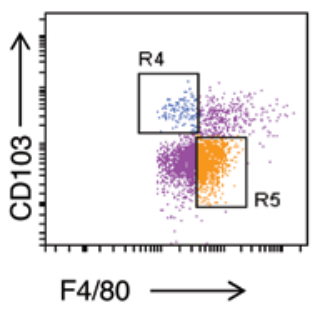

R1, CD11b- DCs R3, CD11C-M\$s $\mathrm{R} 4, \mathrm{CD} 11 \mathrm{~b} * \mathrm{DCs}$

R5, CD11 $\mathrm{c}^{+} \mathrm{M} \Phi \mathrm{S}$

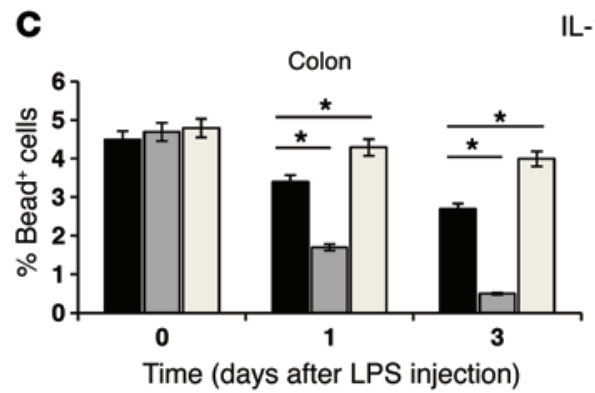

IL-10 KO

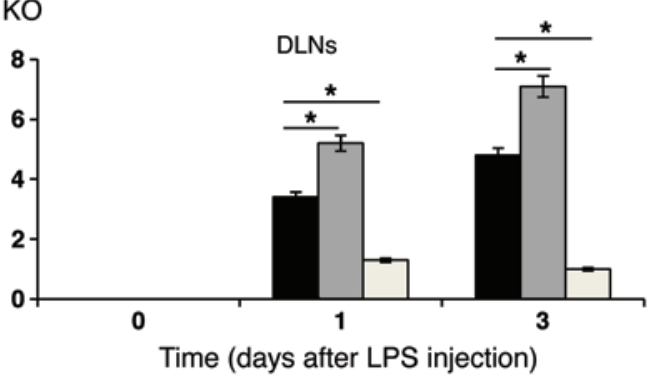

- LPS beads + Ad-GFP

口LPS beads + Ad-hVEGF-C

口LPS beads $+\mathrm{mF} 431 \mathrm{C} 1$

Figure 6. Systemic delivery of Ad-hVEGF-C accelerates bacterial antigen clearance from the inflamed colon to DLNs. (A-C) A single intramucosal injection of carboxylated crimson fluorescent LPS-coated beads was administered on day 5 after the second DSS cycle (chronic inflammation) and on day 21 after the first Ad-hVEGF-C/mF431C1 administration to IL-10-KO mice. Colitic mice treated with Ad-GFP and injected with LPS-coated beads were used as controls. (A) FACS plots showing DCs (R1 and R4) and MФs (R3 and R5) within the bead ${ }^{+}$cell population based on the expression of CD11b (R3), CD11c (R1), F4/80 (R5, gated on R2), and CD103 (R4, gated on R2). (B and C) Clearance of single-bead ${ }^{+}$cells from the inflamed colon (left panels) to the DLNs (right panels) was monitored, and quantification is reported as the mean per group \pm SEM. $n=5$ mice per group. ${ }^{*} P<0.05$ versus LPS-coated beads plus Ad-GFP.

3 days after injection, systemic inhibition of VEGFR3 blocked this effect. As a control, we also determined whether the uptake of coated beads differed between the various cell populations. Coated beads were taken up efficiently by both $\mathrm{CD} 45^{+}$intestinal M $\Phi$ s and DCs 12 hours after the injection (Supplemental Figure 4A).

We then analyzed the phenotype of cleared LPS-coated bead $^{+}$cells in the DLNs 3 days after injection and found that systemic delivery of Ad-hVEGF-C accelerated the migration of both $\mathrm{CD} 11 \mathrm{c}^{-}$and $\mathrm{CD} 11 \mathrm{c}^{+} \mathrm{M} \Phi$ s, but had no effect on CD11b- and $\mathrm{CD}_{11 b^{+}}$DCs (Supplemental Figure 4, B and C). In contrast, blocking of VEGFR3 activation led to the inhibition of both CD11c ${ }^{-}$and $\mathrm{CD} 11 \mathrm{c}^{+} \mathrm{M} \Phi$ migration toward the DLNs without influencing DC clearance (Supplemental Figure 4, B and C), thus demonstrating the specific role of the VEGF-C/VEGFR3 signaling pathway in bacterial antigen clearance by $\mathrm{M} \Phi$ s.

Depleting MTs eliminates protection in VEGF-C-treated mice during chronic experimental colitis. To determine whether the protection seen in VEGF-C-treated mice during disease progression was at least in part the result of VEGF-C-induced MФ activation, clinical parameters, lymph flow, and edema formation were monitored after treatment with clodronate-containing liposomes (CDLs), which have previously been shown to deplete MФs in mouse colons $(23,24,47)$. As shown in Supplemental Figure 5A, CDLs were effective at depleting different $\mathrm{M} \Phi$ subpopulations when compared with PBS-injected mice. Interestingly, while in healthy mice, none of the M $\Phi$ subpopulations expressed VEGFR3, during inflammation, both $\mathrm{CD} 11 \mathrm{c}^{-}$and $\mathrm{CD} 11 \mathrm{c}^{+} \mathrm{M} \Phi$ s upregulated receptor levels on the cell membrane (Supplemental Figure 5, B and C), whereas DCs did not, thus explaining the specific effect of VEGF-C on the biological functions of these cells. While depletion of MФs by CDLs in VEGF-C-treated mice did not affect DSSinduced LV size (Figure 7A), number (Figure 7B, left panel), or lymph flow (Figure 7B, right panel), it did partially restore the phenotype of untreated colitic animals in terms of DSS-induced weight loss (Figure 7C), histological score (Figure 7D and Supplemental Figure 6A left panel), and DAI (Supplemental Figure 6A, right panel). This indicates that the denser and larger VEGF-Cstimulated LV network in the gut is not sufficient per se to resolve inflammation but is accompanied by the role of MФs. We achieved comparable results with IL-10-KO animalas (data not shown). 
A

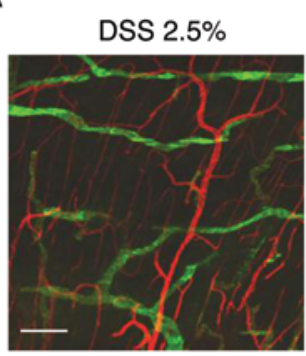

DSS $2.5 \%+$

Ad-hVEGF-C + CDL

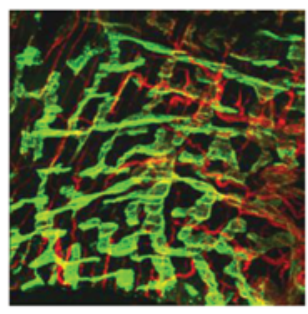

DSS $2.5 \%+$ Ad-hVEGF-C

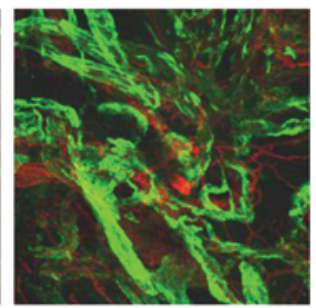

DSS $2.5 \%+$

Ad-hVEGF-C + PBS

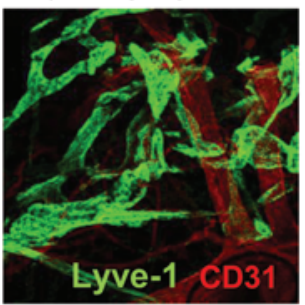

B

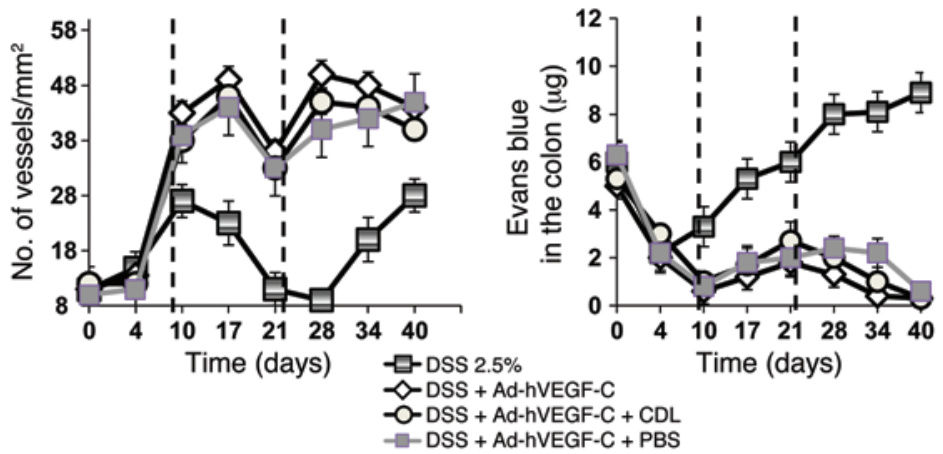

D

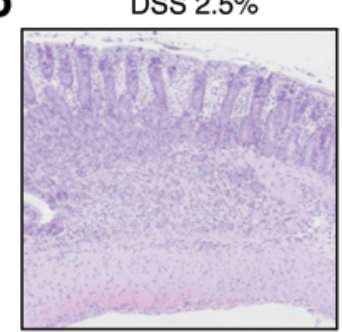

DSS $2.5 \%+$ Ad-hVEGF-C + CDL
-D DSS $2.5 \%$ $\prec$ DSS + Ad-hVEGF-C - - DSS + Ad-hVEGF-C + CDL $\rightarrow$-DSS + Ad-hVEGF-C + PBS
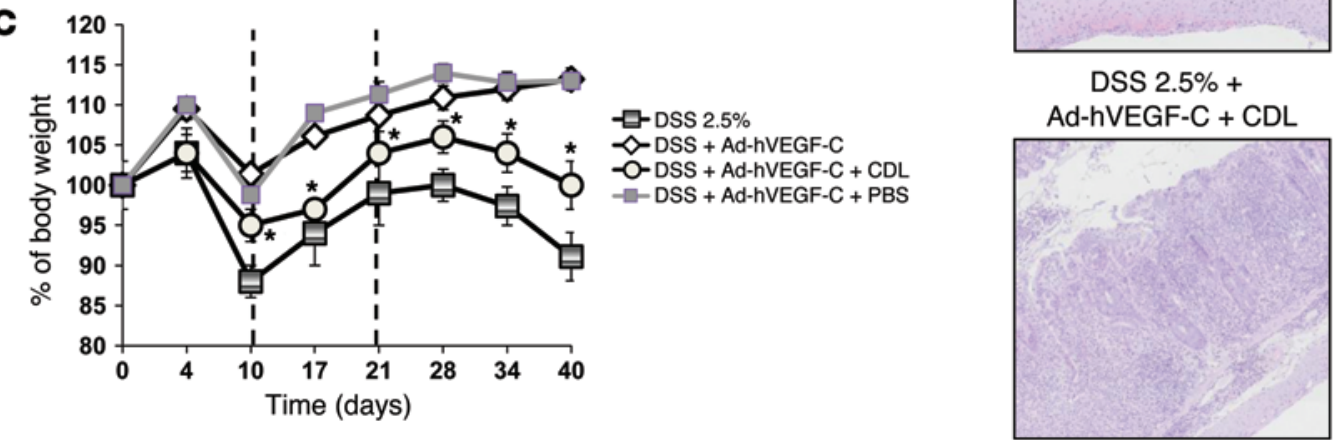

DSS $2.5 \%+$ Ad-hVEGF-C

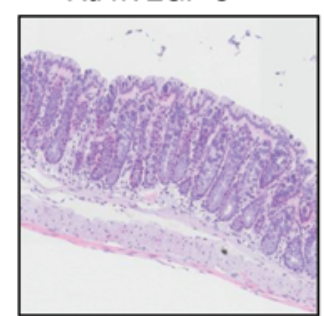

DSS $2.5 \%+$

Ad-hVEGF-C + PBS

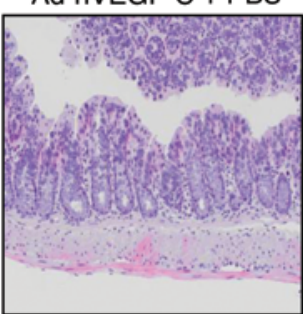

Figure 7. MФ depletion using CDL reduces protection of VECF-C-overexpressing mice during DSS-induced colitis. Mice undergoing 2 cycles of DSS treatment were injected with Ad-hVEGF-C and intrarectally administered CDL or PBS, as described in Methods. (A) Representative images of wholemount staining of distal colons from the indicated groups after the second cycle of DSS (day 28). Scale bar: $75 \mu \mathrm{m}$. (B, left panel) LYVE1+ vessels were counted in 10 regions per section, and the numbers were normalized per total section area expressed in $\mathrm{mm}^{2}$. Results are presented as the mean vessel density per group \pm SEM. (B, right panel) Evans blue was extracted 16 hours after the dye injection from distal colons of comparable weight. Graph shows the total dye remaining in the colon expressed in $\mu$ g. Data represent the mean per group \pm SEM ( $n=4-6 / g r o u p)$. (C and D) CDL administration significantly reduced the protective effect of VEGF-C on the basis of the percentage of body weight (C) and histological examination (D) compared with control mice, which received only DSS plus VEGF-C. Black dashed lines represent the 2 DSS cycles. (D) Representative H\&E-stained images of colons from the indicated groups of mice at the end of the experiment (day 40). Original magnification, $\times 20$. Results in $\mathbf{C}$ are presented as the mean value per group at the indicated time points $\pm \operatorname{SEM}\left(n=4-6 /\right.$ group). ${ }^{*} P<0.05$ versus DSS plus VEGF-C alone.

The VEGF-C/VEGFR3 pathway regulates in vivo and in vitro $M \Phi$ plasticity and activation. Assessing the putative phenotype of VEGF-C-induced MФs in colitic mice, we compared our in vivoderived colonic MФs from VEGF-C-treated animals with those from untreated mice, both in the inflammatory phase of colitis. The release of IL- 6 , TNF- $\alpha$, and IFN- $\gamma$ was significantly lower in the culture of colonic MФs from VEGF-C-induced animals compared with that in the untreated colitic controls, in which cytokine levels were expectedly elevated (Supplemental Figure 6B). In contrast, $\mathrm{M} \Phi$ s isolated from the colons of $\mathrm{MF} 431 \mathrm{C1}$-treated mice revealed an opposite effect (Supplemental Figure 6B). This is consistent with the protective role of the VEGF-C/VEGFR3 pathway in experimental colitis induced by DSS. Interestingly, MФs from VEGF-C-treated mice were alternatively activated, producing increased levels of the M2 markers Ym1, Fizz1, Arg1, and IL-10 compared with those detected in untreated colitic animals (Fig- ure $8 \mathrm{~A}$ ), but these МФs also expressed high levels of typical M1 markers, such as Cox2, MHCII, iNOS, and CD80 (Figure 8B), thus reflecting a hybrid phenotype. When we examined the expression levels of intracellular cAMP, typically increased in this hybrid socalled "resolution-phase" subpopulation (48), we found that they were significantly elevated in MФs isolated from VEGF-C-treated colitic mice compared with the levels detected in their untreated counterparts (Supplemental Figure 6C), confirming the presence of a "resolving" МФ subpopulation. As shown in Figure 8, A and B, and Supplemental Figure 6C, MФs isolated from the colons of $\mathrm{mF} 431 \mathrm{C1}$-treated mice revealed an opposite effect. We obtained similar results with IL-10-KO animals (data not shown).

To assess the putative mechanism by which VEGF-C mediates $\mathrm{M} \Phi$ plasticity and polarized activation, we subjected BM-derived MФs from healthy untreated animals to in vitro M1 (by LPS) and M2 (by IL-4) polarization and VEGF-C stimulation. Our results 
A

M2 markers
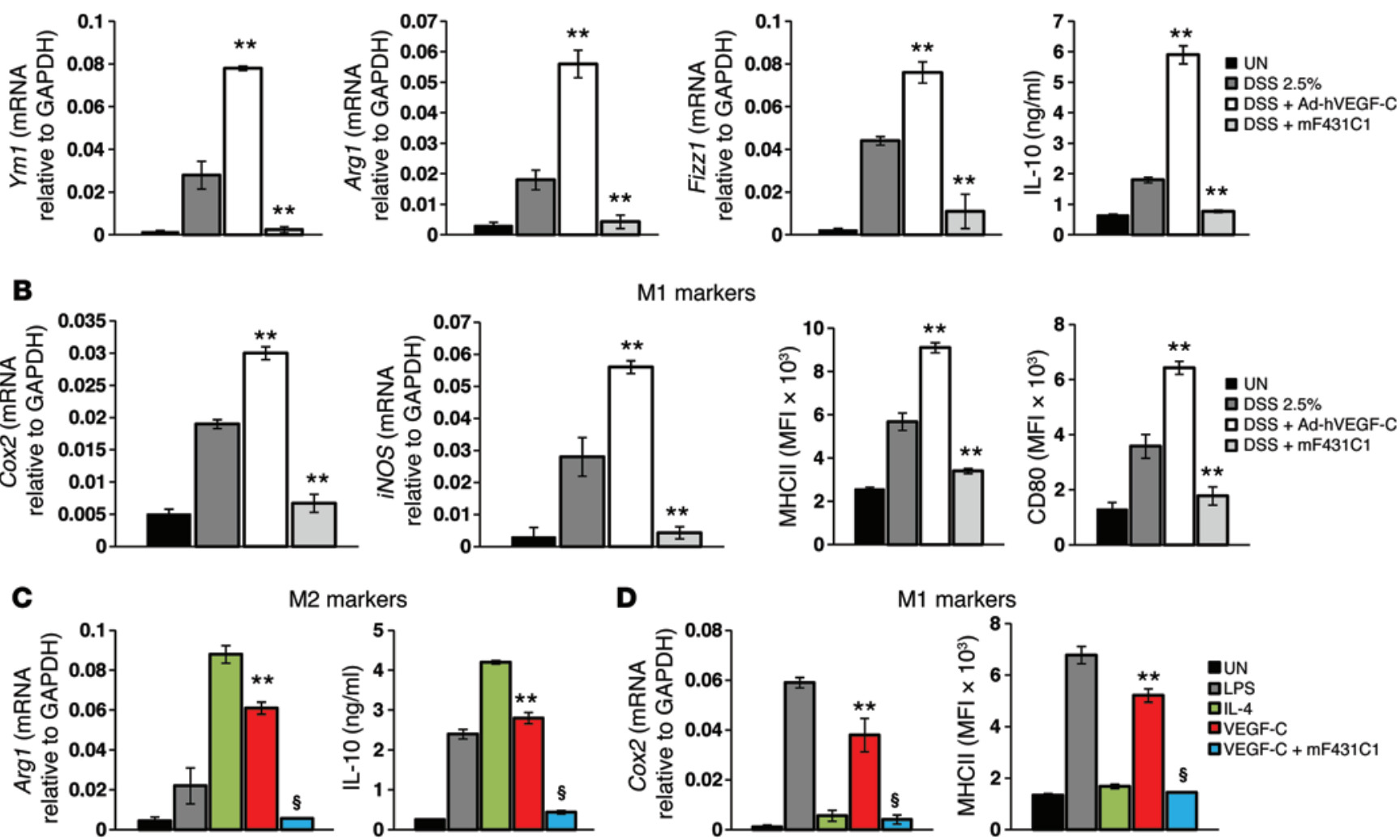

Figure 8. The VEGF-C/VEGFR3 pathway regulates in vivo and in vitro MФ plasticity and activation. (A and B) Mice undergoing 2 cycles of DSS treatment were injected with Ad-hVEGF-C or anti-VEGFR3 Ab. Colonic expression of M2 (A) and M1 (B) M $\Phi$-associated genes was examined by real-time PCR analysis for Ym1, Arg1, Fizz1, Cox2, and iNOS; by ELISA for IL-10; and by FACS for MHCII and CD80 after 24 hours in vitro. Data represent the means \pm SEM per group ( $n=4-6$ /group). (A and B) ${ }^{* *} P<0.01$ versus DSS alone. (C and D) BM-derived MФs were cultured and stimulated in vitro with LPS (M1), IL-4 (M2), VECF-C, and VEGF-C plus mF431C1 for 24 hours. Expression of the indicated genes was then examined by real-time PCR, ELISA, or FACS. Data represent the means \pm SEM of 3 independent experiments. ${ }^{*} P<0.01$ versus untreated $(U N) ;{ }^{\circledR} P<0.001$ versus VEGF-C alone.

showed that VEGF-C alone strongly increased both M1 and M2 gene expression, almost comparable to that seen in control cells stimulated with LPS or IL-4 alone (Figure 8C and Supplemental Figure 6D); these outcomes were abolished upon treatment with the anti-VEGFR3 Ab (Figure 8C and Supplemental Figure 6D). Overall, these data confirm the induction of a unique in vitro mixed M1-M2 subpopulation, as observed in vivo, and demonstrate a direct effect of the VEGF-C/VEGFR3 pathway not only on $\mathrm{M} \Phi$-mediated bacterial antigen clearance (Figure 6) but also on $\mathrm{M} \Phi$ plasticity and polarization (Figure 8).

Resolving MФs mediate the protective role of the VEGF-C/ VEGFR3 pathway during chronic experimental colitis in a STAT6dependent manner. At the transcriptional levels, IL-4-induced differentiation of alternatively activated $M \Phi s$ and the expression of genes such as MHC class II are mediated by the transcription factor STAT6 $(49,50)$. For this reason, we asked whether VEGF-C induced the expression and/or activation of STAT6 in the resolution-phase subpopulation of MФs (rMФs). Upon VEGF-C treatment, the amount of total STAT6 remained comparable to that in the untreated cells, whereas increased levels of phosphorylated STAT6 (p-STAT6) were detected, in both in vivo- (Figure 9A, left panel) and in vitro BM-derived differentiated MФs (Figure 9A, right panel). This effect was inhibited by $\mathrm{mF} 431 \mathrm{C} 1$ (Figure 9A), showing that the VEGF-C/VEGFR3 pathway directly modulates STAT 6 activation during experimental colitis and in cultured BMderived MФs. In addition, inhibition of STAT6 in VEGF-C-treated cells using a specific siRNA abolished the effect of this growth factor on the expression of M2- (Figure 9B and Supplemental Figure 7A) and M1- (Figure 9C and Supplemental Figure 7B) associated genes and on $\mathrm{M} \Phi$ migration through matrigel-coated filters (Supplemental Figure 7C), whereas the scramble control did not, demonstrating that the VEGF-C/VEGFR3 pathway directly controls M $\Phi$ plasticity and migration in a STAT6-dependent manner. Finally, since we demonstrated that the protection seen in VEGF-Ctreated mice during disease progression was at least in part the result of resolving $М \Phi$ activation, we investigated the mechanism through which this pathway confers protection during DSS-induced colonic inflammation. For this purpose, we injected in vitro VEGF-C-induced $\mathrm{rM} \Phi$ s isolated from the BM of GFP mice into the rectal mucosa of colitic mice every 96 hours and monitored the clinical parameters at different time points for the entire experiment (Figure 9D). Notably, 48 hours after the injection, we still found $\mathrm{GFP}^{+} \mathrm{M} \Phi$ s in the colons of colitic mice, while we no longer detected these cells after 96 hours (data not shown), thus allowing us to determine the injection intervals. These animals showed ameliorated clinical parameters in terms of body weight (Figure 9E), 
A

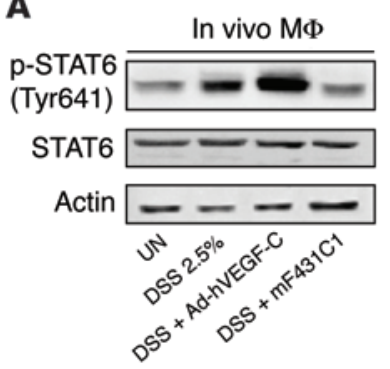

B

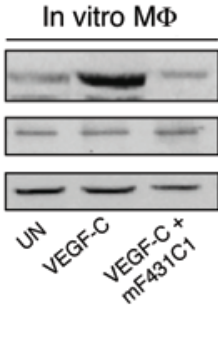

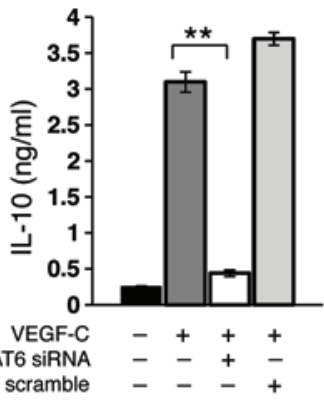

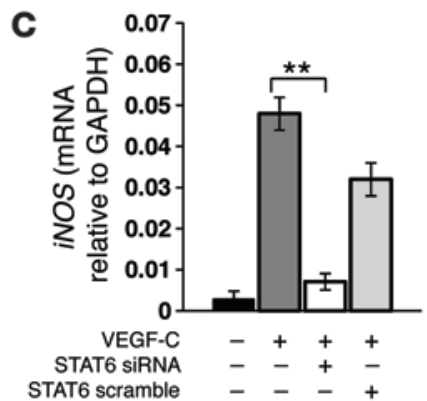

D

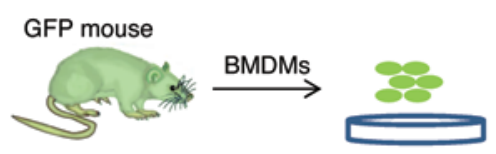

In vitro differentiated:

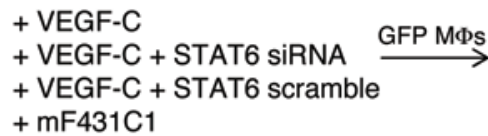

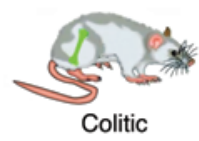

$\longrightarrow$ parameters
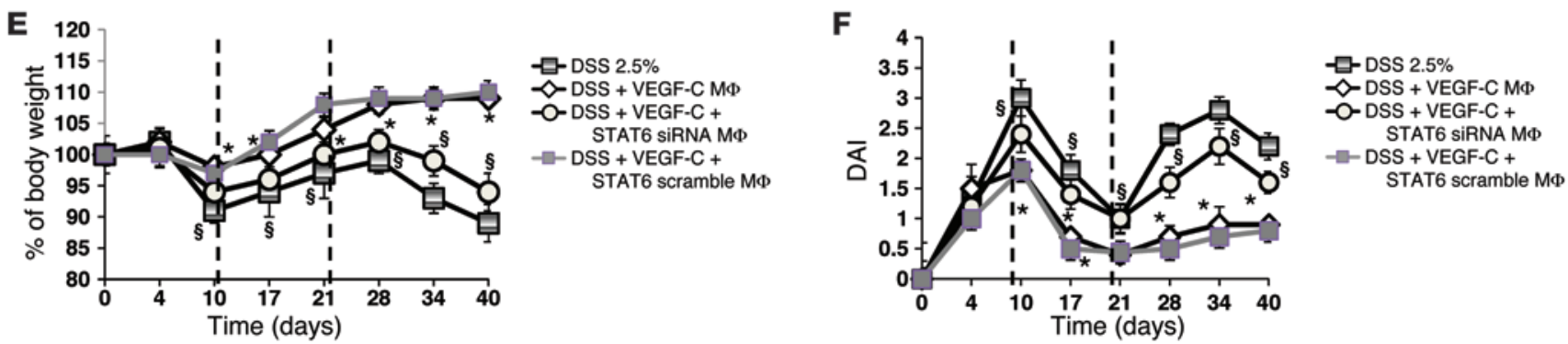

Figure 9. Resolving MФs mediate the protective role of the VEGF-C/VEGFR3 pathway during chronic experimental colitis in a STAT6-dependent manner. (A) Mice undergoing 2 cycles of DSS treatment were injected with Ad-hVEGF-C or anti-VEGFR3 Ab. Colonic expression of p-STAT6 and total STAT6 was evaluated by Western blotting in MФs after 4 hours in culture (in vivo) and in BM-derived differentiated MФs (in vitro). Actin was used as a loading control. (B and C) BM-derived MФs were cultured, transfected with a STAT6-specific siRNA or a STAT6 scramble, and stimulated in vitro with VEGF-C for 24 hours. Expression of the indicated genes was then examined by ELISA (B) or real-time PCR (C). Data represent the means \pm SEM of 3 independent experiments. ${ }^{* *} P<0.01$. (D-F) GFP plus BM-derived MФs (BMDMs) were cultured, transfected with a STAT6-specific siRNA or a STAT6 scramble, and stimulated in vitro with VEGF-C or mF431C1 (D). Cells were then injected into the rectal mucosa of DSS-treated mice ( $n=4 /$ group), and clinical parameters such as body weight $(\mathbf{E})$ and DAI (F) were evaluated. Black dashed lines represent the 2 DSS cycles. Results are presented as the mean value per

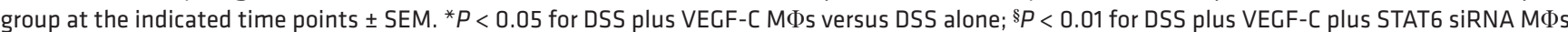
versus DSS plus VEGF-C MФs.

DAI (Figure 9F), and colon length (Supplemental Figure 7D) when compared with those of the untreated animals. This protective role was abolished by STAT6-silenced VEGF-C-induced MФs (Figure 9, E and F, and Supplemental Figure 7D), demonstrating, for the first time to our knowledge, one of the mechanisms of action through which VEGF-C/VEGFR3 signaling abates the severity of experimental colitis.

\section{Discussion}

In this study, we report that the specific promotion of LV function limits experimental chronic intestinal inflammation in mice that is mediated by a unique $\mathrm{M} \Phi$ polarization and activation and modification of the tissue cytokine milieu. These novel findings are important because, although the precise pathophysiology of IBD is unknown, $\mathrm{LV}$ obstruction and dysfunction are long-recognized features observed in humans with this disease $(15,16)$, and normalizing gut lymphatics with prolymphangiogenic factors such as VEGF-C may improve disease activity. Consistent with altered lymphatic contractile function reported in an animal model of acute intestinal inflammation (19), intestinal lymphatics are known to be grossly abnormal in the gut of $\mathrm{CD}$ patients (10). Moreover, mucosal and submucosal edema is a typical feature of IBD tissues despite early abundant lymphatic vasculature. Nevertheless, only limited information is available on the clearance capacity of LVs in IBD (17), and some of the lymphatics might be immature or nonfunctional, as appears to be the case in tumor-associated lymphangiogenesis $(51,52)$. Recent work by Tammela et al. (53) demonstrated that by stimulating the VEGFR3 tyrosine kinase by treatment with the lymphangiogenic factor VEGF-C, it is possible to regenerate functional collecting LVs in mice following lymph node (LN) dissection. As VEGFR3 activity plays important roles in both the maintenance and survival of lymphatic endothelial cells (LECs) and vascular integrity, decreased VEGFR3 expression or signaling might alter many lymphatic functions, leading to increased gut injury. Indeed, antilymphatic treatment with anti-VEGFR3 antibodies aggravated inflammation and submucosal edema, increasing leukocyte infiltration in an animal model of IBD (13). In the current study, we demonstrate activation of the VEGF-C/ VEGFR3 pathway in the actively inflamed mucosa of patients with IBD. Except for VEGF-D, another VEGFR3 ligand whose levels remain stable in actively inflamed intestinal tissues, expression of both VEGF-C and VEGFR3 was significantly increased in biopsy 
specimens from inflamed bowel segments when compared with those in controls, a finding compatible with inflammation-associated lymphangiogenesis. Similarly, we found that the expression of VEGF-C and its receptor, both expressed in colon tissue under physiologic conditions, was markedly enhanced during experimental colitis in 2 different animal models: the chronic DSS and the IL-10-KO models, $(54,55)$. Thus, these models offer a good platform for manipulating the VEGF-C/VEGFR3 pathway and verify its effect on the course of experimental intestinal inflammation. Indeed, we demonstrated that systemic inhibition of VEGFR3 blocked lymphangiogenesis, reducing both area density and LV dimension and growth, while significantly increasing inflammatory edema formation and inhibiting disease resolution, as also observed by Jurisic et al. (13). In contrast, lymphatic drainage was enhanced by systemic delivery of VEGF-C, which in turn significantly induced LV density and proliferation, improving intestinal inflammation. The drainage-promoting function of VEGF-C in our models is in agreement with recent findings showing that the inhibition of VEGF-C by soluble VEGFR3 (sVEGFR3) significantly decreased lymph flow in a model of bacterial skin inflammation (56) and that genetic overexpression of sVEGFR3 in the skin of mice resulted in a lymphedema-like phenotype (57). Conversely, the application of VEGF-C or tumor-derived VEGF-C enhances lymphatic function and flow, respectively $(53,58-61)$.

As reported by others $(62,63)$, we did not detect any changes of the blood vasculature in VEGF-C-treated mice. Therefore, the improvement of experimental colitis mediated by systemic delivery of Ad-hVEGF-C is likely not caused by effects on blood vessels. Interestingly, the enhanced lymphatic drainage by VEGF-C was observed in combination with increased inflammatory cell mobilization and bacterial antigen clearance, all LV functions that we found to be inhibited by VEGFR3 blockade. VEGF-Cdependent antigen clearance was a $M \Phi$-specific (both CD11c and $\mathrm{CD}_{11^{+}}$) effect, supported by the fact that these cells express VEGFR3 on the cell membrane during inflammation, whereas DCs do not. Such results suggest a potential role for VEGFR3 signaling in immunity by mediating antigen-presenting cell (APC) trafficking through $\mathrm{M} \Phi$ recruitment. The most intriguing and novel finding in this study is that the protection we observed in VEGF-C-treated mice during disease progression was not only a consequence of increased lymphangiogenesis and enhanced lymphatic flow and function, including mobilization of inflammatory cells and bacterial antigen clearance, but was also a result of a previously unknown direct VEGF-C-induced M $\Phi$ activation through STAT6 signaling. The M $\Phi$ has long been considered part of the destructive force behind IBD as a result of its involvement in regulating inflammation through the production of inflammatory cytokines and chemokines (64-66). When activated, MФs can be functionally divided into the classically activated, or M1, type and the alternatively activated, or M2, type in response to the different stimuli of the local microenvironment (67). Our data show that administration of VEGF-C activates a hybrid M $\Phi$ phenotype, with a direct effect on cell migration and expression of both M1 and M2 markers, with opposite effects observed upon blocking of VEGFR3. This specific subpopulation has already been observed in a peritoneal model of inflammation, in which the socalled $\mathrm{rM} \Phi$ s expressed a unique mixed M1-M2 phenotype, with a role in mediating post-resolution innate lymphocyte repopulation and restoring tissue homeostasis $(48,68)$. We show that adoptive transfer of VEGF-C-differentiated resolving МФs in colitic mice inhibited the clinical parameters of DSS-induced chronic colitis, in agreement with reports demonstrating that in vitro-differentiated monocytes have antiinflammatory properties or enhance the resolution of inflammation $(23,69)$. In addition, we discovered that the polarization and resolving actions of MФs are mediated by the VEGF-C/VEGFR3 pathway through STAT6 activation. This is intriguing, because some reports suggest that there may be a dysregulation of STAT 6 signaling in the ungoverned immune response associated with colitis (70-72), and this transcription factor plays a regulatory role in the pathogenesis of IBD (73),

Although not assessed in our study, it would be of interest to investigate the in vivo beneficial effects of VEGF-C-activated MФs in terms of $\mathrm{T}$ cell immunosuppression and the potential role of DLN lymphangiogenesis in the control of intestinal inflammation $(74,75)$. The increased drainage capacity and resolution of chronic intestinal inflammation might, at least in part, be mediated by enhanced VEGF-C-induced DLN lymphangiogenesis in addition to the increased lymphangiogenesis we observed in the inflamed colon itself. Nevertheless, we cannot fully exclude the possibility that other VEGF-C activities, including other direct effects on the inflamed lymphatic vasculature, are involved in the antiinflammatory properties of VEGF-C.

In conclusion, our study provides the first proof of concept to our knowledge that it may be possible to treat chronic gastrointestinal inflammatory disorders by stimulating LV functions to promote drainage and bacterial antigen clearance, together with adaptive immunity, effects achievable through modulation of the VEGF-C/VEGFR3 pathway. In fact, increased VEGF-C expression levels found in mucosal extracts of patients with IBD could be insufficient to promote proper lymphatic function and MФ-polarized activation and migration. VEGF-C gene therapy can induce growth of new LVs in the skin of Chy mice, suggesting that some forms of lymphedema might be treated by similar approaches in humans (59). In addition, use of VEGF-C plasmid in rabbit ear and mouse tail lymphedema models results in amelioration of lymphatic function and alleviation of the signs of lymphedema (61). Thus, these and our own observations support the potential use of lymphangiogenic growth factors as a novel therapeutic approach for the treatment of IBD and other chronic inflammatory diseases.

\section{Methods}

\section{Patients}

Intestinal tissues from involved areas were obtained from surgical specimens of patients with CD and UC. Healthy tissues from the intestine of patients admitted for bowel resection due to colon cancer, polyps, or diverticulosis were used as controls. Specimens were formalin fixed and paraffin embedded or frozen in Cryoblock Compound (DiaPath) on dry ice and stored at $-80^{\circ} \mathrm{C}$.

\section{Mice}

Mice used in this study were male or female C57BL/6 (Charles River Laboratories) or Il1O ${ }^{\text {tmICgn }}$ (IL-10-KO) and IL-10 WT mice on a C57BL/10 background and were provided by Giovanni Pietrogrande 
(Ospedale San Raffaele, Milan, Italy). All animals were maintained under pathogen-free conditions.

\section{Induction and evaluation of colonic inflammation}

As reported previously (54), 3- to 4-week-old IL-10-KO mice consistently develop colitis at 10 to 12 weeks of age when transported from the ultrabarrier facility (UBF) to conventional housing. Clinical signs of inflammation are diarrhea, perianal ulceration, intestinal bleeding, and occasional rectal prolapse $(76,77)$. Intestinal histopathology of IL-10$\mathrm{KO}$ mice shares some features with human $\mathrm{CD}$. The inflammatory process is indeed characterized by focal transmural inflammation, mucosal proliferation, focal ulceration, and extensive infiltration of lymphocytes, plasma cells, MФs, and scattered neutrophils. IL-10 WT animals were used as controls. A slightly modified version of the previously described DAI (54) was calculated by scoring 1 point for the appearance of each of the following: occult fecal blood, as determined on a Hemoccult SENSA Card (Beckman Coulter), rectal prolapse, diarrhea, or more than $5 \%$ weight loss. A 5-point (0-4) DAI was thus obtained.

For the DSS-induced chronic colitis model, 8- to 12-week-old female mice were subjected to 2 oral cycles of $2.5 \%$ DSS (molecular mass, $40 \mathrm{kDa}$; MP Biomedicals), each cycle characterized by 10 days of DSS exposure followed by 11 days of filter-purified water (78). The control mice group received water without DSS. In this model, the DAI scale is based on the evaluation of different parameters characterizing experimental colitis induction and progression. Body weight, presence of gross blood in the feces, and stool consistency were recorded at the indicated time points (Figure 2 and Supplemental Figure 2). According to the criteria proposed by Cooper et al. (79), the DAI was determined by scoring changes in: weight loss $(0=$ none, $1=1 \%-5 \%, 2=5 \%-10 \%$, $3=10 \%-20 \%, 4=>20 \%)$; stool consistency ( $0=$ normal, $2=$ loose, 4 = diarrhea); and rectal bleeding ( 0 = normal, 2 = occult bleeding, 4 = gross bleeding). A 5-point (0-4) DAI was thus obtained.

For both models, mice were sacrificed at the indicated time points (Figure 2), and grading of intestinal inflammation was confirmed histologically in a blinded fashion, as previously described $(8,80)$. Colons of colitic mice and healthy controls were evaluated for histological analyses using 2- $\mu \mathrm{M}$ paraffin-embedded sections, stained with hematoxylin (Dako) and eosin (Diapath). A blinded pathologist evaluated the degree of inflammatory cell infiltration and mucosal damage, as previously described (8). In parallel experiments, colonic biopsies were embedded in Cryoblock Compound for immunofluorescence studies or immediately frozen on dry ice for Western blot analyses.

VEGFR3 Ab blockade. Fifteen-week-old IL-10-KO and WT mice (not shown) with fully established colitis, as determined by weight loss, diarrhea, bloody stools, and rectal prolapse, were given i.p. injections of an anti-VEGFR3 Ab, mF431C1 (clone 31C1, $800 \mu \mathrm{g} / 100 \mu \mathrm{l}$ of PBS per mouse; ImClone Systems) (27), or PBS alone 3 times per week for a period of 5 weeks. In the DSS model of colitis, $\mathrm{mF} 431 \mathrm{C} 1$ or PBS was administered i.p. 3 times per week for the entire experiment, starting from the first day of DSS administration.

Ad-hVEGF-C administration. Fourteen-week-old IL-10-KO and WT mice (not shown) with fully established colitis were given i.v. injections of an engineered Ad-hVEGF-C or a control virus encoding GFP (Ad-GFP) $\left(5 \times 10^{8}\right.$ PFU per mouse; ViraQuest). A second dose of Ad-hVEGF-C was administered 28 days after the first injection. In the DSS model of colitis, Ad-hVEGF-C or Ad-GFP was administered i.v. the first day of DSS administration, with a second injection 28 days later. Mice were killed at the indicated time points (Supplemental Figure 3), and mucosal extracts were analyzed by Western blotting to determine VEGF-C expression and inhibition of VEGFR3 activity. All experiments were performed twice, using 4-8 mice per experimental group. Adenoviral preparation was free from contamination with helper viruses, bacteria, or LPS.

\section{Immunostaining of human and murine colonic tissues}

Human. To quantify LV density, the number of podoplanin ${ }^{+}$LVs was assessed in inflamed areas of surgical specimens from patients with CD and UC (IBD). Normal areas of the intestine from patients admitted for bowel resection due to colon cancer, polyps, or diverticulosis were used as controls (NL). Tissues were fixed in $10 \%$ buffered formalin, processed, and stained with hematoxylin and eosin or immunostained with specific Abs. Sections (2- $\mu$ m-thick) of formalin-fixed, paraffin-embedded tissues were deparaffinized in Bioclear (Bioptica) and rehydrated in a descending ethanol series. Following antigen retrieval by heating for 10 minutes in a microwave with EDTA buffer ( $\mathrm{pH} 8.0$; $0.25 \mathrm{mM})$, sections were incubated for 20 minutes with peroxidase 1 solution (Biocare Medical) to quench endogenous tissue peroxidase. Tissue slides were then incubated for 60 minutes at room temperature (RT) in a humid chamber with mouse anti-human podoplanin mAb (clone D2-40, 1:100; ABD Serotec), and staining was completed using the Biocare Medical HRP-conjugated mouse detection kit and DAB chromogen as a substrate, according to the manufacturer's instructions. Last, sections were counterstained with CAT Hematoxylin (Biocare Medical) for 5 minutes. After staining, the slides were dehydrated through graded alcohols and mounted with a coverslip using Eukitt Quick Hardening Mounting Media (Fluka Analytical, Sigma-Aldrich). Negative controls, with omission of the primary incubation, were also included. The slides were analyzed randomly by 2 of the authors blinded to the clinical data.

LV density was quantified in the submucosal and subserosal layers, both areas that are richly vascularized by lymphatic capillaries. Only vessels stained with podoplanin and visible lumina were considered countable, because small nerve fibers and fibroblasts also expressed podoplanin (81) and potentially could be confused with collapsed lymphatic capillaries. The mean vessel density of 10 areas with an elevated number of LVs in the $\times 20$ magnification objective field were assessed separately using VS-ASW software (Olympus).

Mouse. To achieve labeling of LECs undergoing DNA synthesis, untreated and treated mice subjected to the DSS protocol or WT and IL-10-KO treated animals received an i.p. injection of $100 \mathrm{mg} / \mathrm{kg}$ BrdU 2 hours prior to sacrifice. Formalin-fixed, paraffin-embedded colon sections $(2-\mu \mathrm{m})$, at the indicated time points (Figure 4$)$, were double-stained for LYVE1 and BrdU after antigen retrieval for $30 \mathrm{~min}$ utes at $98^{\circ} \mathrm{C}$ in citric buffer $(10 \mathrm{mM}, \mathrm{pH}$ 6.0). Following endogenous peroxidase blocking with peroxidase 1 solution for 20 minutes at RT, tissues were washed in TBS containing 0.05\% Tween-20 instead of PBS to reduce alkaline phosphatase aspecific staining. Upon incubation with the goat anti-mouse LYVE1 (1:100; R\&D Systems) primary $\mathrm{Ab}$ for 90 minutes at RT, sections were stained with rat anti-BrdU Ab (1:600; AbD Serotec) for 40 minutes at $4^{\circ} \mathrm{C}$ (Biocare Medical). A rat HRP polymer kit (Biocare Medical) was used to detect BrdU using DAB (Biocare Medical) as chromogen, followed by the Goat AP-Polymer Kit (Biocare Medical) to detect LYVE1 with Warp Red (Biocare Medical) as an alkaline phosphatase substrate. Tissue sections were then counterstained with CAT Hematoxylin (Biocare Medical), dried overnight in a 
$37^{\circ} \mathrm{C}$ oven, and mounted in Eukitt Quick Hardening Mounting Media. Images were acquired with a VS120 Dot Slide System (Olympus), and BrdU-LYVE $1^{+}$cells were counted in consecutive and randomly selected fields ( $n=10$ fields/section for each group) at the indicated time points shown in Figure 4 using VS-ASW software.

\section{Morphometric and immunofluorescence intensity measurements}

Morphometric analysis of colon whole mounts was performed by $3 \mathrm{D}$ visualization of LVs. Images were taken as a $z$ series stack using a $\times 10$ objective and analyzed by Imaris Bitplane software, thus allowing visualization of the LVs in the submucosal and serosal-muscular layers. For each colon analyzed ( $n=5-8$ mice each experimental group), $\mathrm{LV}$ density and size were measured in 10 randomly chosen regions covered by vessels (each $1.0 \mathrm{~mm}^{2}$ in area) and were represented as the average number of vessels per area $\left(\mathrm{mm}^{2}\right)$ and the mean value of diameters $(\mu \mathrm{m})$, respectively.

The number of LYVE1-, VEGFR3-, or CD31+ vessels in single- and double-stained human and murine colon tissues was established by stereological point counting of 10 regions per section (mucosal, submucosal, and serosal layers), each $1.5 \mathrm{~mm}^{2}$ in area, using FluoView software (Olympus). Values are expressed as the mean number of positive vessels per $\mathrm{mm}^{2}$.

Analysis of human and mouse VEGFR3 and LYVE1 double staining was performed as described by Tammela et al. (36), with slight modifications. In brief, murine colon sections were double stained with rabbit anti-LYVE1 (1:400; Abcam) and goat anti-VEGFR3 (1:50; R\&D Systems) primary Abs, while staining of human colon sections is described above. Fluorescent images were acquired at a constant exposure time at $\times 20$ magnification on a laser-scanning confocal microscope (FluoView FV1000). Colons stained with secondary Abs alone were used to set the exposure time. Vascular structures with a lumen were analyzed with FluoView software, and all images were within a linear intensity range of 0 to 6,231 . To exclude nonspecific staining, structures less than $8 \mu \mathrm{m}$ $(1 \mu \mathrm{m}=6.8$ pixels $)$ in diameter were excluded. To calculate mean vessel intensity, the sums of pixel intensities per vessel were divided by the total vessel area $\left(\mu \mathrm{m}^{2}\right)$. The vessel mean fluorescence intensity (MFI) from 5 to 10 images per colon ( $n=5-8$ mice per experimental group) was averaged and compared between the treated and control groups.

\section{Assay for inflammatory cell migration from the site of inflammation to the DLNs}

$\mathrm{GFP}^{+}$mice were injected i.p. with $1 \mathrm{ml} 3 \%$ thioglycolate (Sigma-Aldrich) in saline, and inflammatory cells were collected 3 days later by peritoneal washing with ice-cold DMEM culture medium. Approximately $10^{6}$ $\mathrm{GFP}^{+}$inflammatory cells in $10 \mu \mathrm{l}$ PBS were adaptively transferred by injection into the noninflamed and inflamed rectal mucosa of untreated and treated mice subjected to the DSS protocol (at day 5 after the second DSS cycle) or into the noninflamed and inflamed rectal mucosa of WT and IL-10-KO treated animals (at day 21 after the first administration of Ad-hVEGF-C). Twelve hours later DLNs, including the mesenteric and lumbar LNs, were collected, frozen in Cryoblock Compound and sectioned for immunofluorescence, and a cell suspension was obtained by smashing through a 70- $\mu \mathrm{m}$ filter (BD Falcon) with a 1-ml syringe plunger and washed with $10 \mathrm{ml}$ RPMI medium supplemented with $10 \%$ FCS and $20 \mathrm{mM}$ HEPES. Frozen sections of DLNs were fixed and blocked as described above and incubated with rabbit anti-LYVE1 primary $\mathrm{Ab}$ (1:400; Abcam) for 1 hour at RT. Sections were then incubated for
30 minutes at RT with Alexa Fluor 647-conjugated anti-mouse (1:1,000; Invitrogen), followed by incubation with DAPI (1:25,000; Invitrogen). DLN tissues were then mounted with ProLong Gold mounting medium (Invitrogen) and analyzed with a laser-scanning confocal microscope (FluoView FV1000). Images were acquired with FluoView software, and $\mathrm{GFP}^{+}$cells were counted in the entire section and expressed as the mean number of $\mathrm{GFP}^{+}$cells $/ \mathrm{mm}^{2}$. In the FACS data, the histogram numbers represent the percentage of $\mathrm{GFP}^{+}$cells.

\section{Lymph flow assessment using Evans blue dye}

Ten micrograms of Evans blue dye (Sigma-Aldrich) in $10 \mu \mathrm{l}$ of PBS was injected into the rectal mucosa of anesthetized untreated and treated mice subjected to the DSS protocol or WT and IL-10-KO treated animals ( $n=5$ mice per experimental group) using a Hamilton syringe. After 16 hours, mice were sacrificed, and Evans blue dye was extracted from distal colons of comparable weight by incubating the tissues at $55^{\circ} \mathrm{C}$ overnight in formamide (Sigma-Aldrich). The background-subtracted absorbance was measured with a Versamax microplate reader (Molecular Devices) at a wavelength of 620 to $740 \mathrm{~nm}$. The concentration of dye in the extracts was calculated from a standard curve of Evans blue in formamide and presented as the absolute amount of dye that remained in the colons.

\section{Monitoring bacterial antigen clearance}

Coating of beads with LPS. Carboxylated crimson fluorescent $1-\mu \mathrm{m}$ beads (Molecular Probes) were coated with LPS from E. coli 0127:B8 (Sigma-Aldrich), according to the manufacturer's instructions. All steps were performed in glass tubes. LPS was dissolved at a concentration of $2.5 \mathrm{mg} / \mathrm{ml}$ in $50 \mathrm{mM}$ MES buffer (pH 6) and incubated for 15 minutes at RT with $5 \mathrm{ml}$ of a $2 \%$ aqueous suspension of microspheres. 1-Ethyl-3-(3-dimethylaminopropyl) carbodiimide was then added at a concentration of $5 \mathrm{mg} / \mathrm{ml}$. Upon adjustment of the $\mathrm{pH}$ to $6.5 \mathrm{using} 1 \mathrm{M}$ $\mathrm{NaOH}$, the solution was incubated for 2 hours at RT on a rocker. Coated beads were washed 3 times and resuspended in PBS with 1\% BSA.

Intramucosal administration of LPS beads. Untreated and treated mice subjected to the DSS protocol or WT and IL-10-KO treated animals ( $n=5$ per experimental group) were intramucosally administered $50 \mu \mathrm{l}$ LPS-coated bead or uncoated bead (not shown) solution containing $3.6 \times 10^{9}$ beads using an insulin U-100/0.3 ml syringe (Terumo). Three days after injection, the animals were sacrificed, and the colons and DLNs were collected for analysis of both phenotype and migration patterns. For colons, tissues were cut and washed in HBSS buffer containing 5\% FCS, $20 \mathrm{mM}$ HEPES, $100 \mathrm{Unit} / \mathrm{ml}$ penicillin, and $100 \mu \mathrm{g} / \mathrm{ml}$ streptomycin. DTT $(1 \mathrm{mM})$ was then added for 2 minutes to remove mucous. Colons were cut in small pieces and incubated for 1 hour at $37^{\circ} \mathrm{C}$ in an enzymatic solution containing 120 Units of collagenase type II (Worthington), $5 \mathrm{mM} \mathrm{CaCl}_{2}$ (VWR International), and 20-50 $\mu \mathrm{g} / \mathrm{ml}$ DNAse I (Roche). Tissues were then filtered and washed in RPMI medium supplemented with 10\% FCS and 20 mM HEPES. DLNs were smashed through a $70-\mu \mathrm{m}$ filter (BD Falcon) with a 1-ml syringe plunger and washed with $10 \mathrm{ml} \mathrm{RPMI} \mathrm{medium} \mathrm{supplemented} \mathrm{with} 10 \%$ FCS and $20 \mathrm{mM}$ HEPES. The uptake of beads by MФs and DCs and the effects on their phenotype were analyzed by flow cytometry. For this purpose, cells were centrifuged and resuspended in PBS supplemented with 0.5\% BSA and $0.005 \% \mathrm{NaN}_{3}$ (FACS buffer) and incubated for 30 minutes on ice with the following Abs: PE-conjugated anti-CD103 (clone M290;1:100; BD Pharmingen), PerCP-conjugated anti-CD45 (clone 30-F11; 1:100; 
BD Pharmingen), PE-Cy7-conjugated anti-F4/80 (clone BM8; 1:100), allophycocyanin-conjugated anti-CD11c (clone HL3;1:100), and Pacific blue-conjugated anti-CD11b (clone M1/70;1:100) (all from eBioscience unless otherwise noted). MФ and DC subpopulations were assessed according to the expression levels of these markers, as shown in Figure 6A. Stained cells were analyzed using a FACSCanto (BD Biosciences), and data were analyzed using FACSDiva software (BD Biosciences).

\section{Adoptive transfer of VEGF-C-induced MФs in colitic mice}

$\mathrm{BM}$-derived MФs were flushed from the femurs and tibia of 7- to 12-week-old GFP ${ }^{+}$mice and treated as described in the Supplemental Information. Fully differentiated monocyte-derived MФs were transfected with STAT6 or scramble siRNA (all from Santa Cruz Biotechnology Inc.) using Lipofectamine 2000 reagent (Invitrogen) according to the manufacturer's instructions. Seventy-two hours after transfection, cells were either left untreated or were stimulated with VEGF-C (100 ng/ml; R\&D Systems) or mF431C1 $(300 \mu \mathrm{g} / \mathrm{ml}$; ImClone) for 24 hours. МФs were then retrieved, and $1.0 \times 10^{6}$ cells were injected into the rectal mucosa of anesthetized DSS-treated mice ( $n=4-6 /$ group) using an insulin U-100/0.3 ml syringe (Terumo), starting from day 4 and then every 4 days for the entire experiment. Clinical parameters such as body weight, DAI, and colon length were evaluated as described above.

\section{Statistics}

Statistical analyses were performed using the statistical functions of Microsoft Excel 2010. The data represent the means \pm SEM, as indi- cated, and significant differences between the means were analyzed with a 2-tailed, unpaired Student's $t$ test. A $P$ value of less than or equal to 0.05 was considered statistically significant.

\section{Study approval}

Human studies were approved by the ethics committee of the Istituto Clinico Humanitas (Rozzano, Italy). Procedures involving mice conformed to institutional guidelines in agreement with national and international law and were approved by the ethics committees of the Istituto Clinico Humanitas and Ospedale San Raffaele (Milan, Italy).

\section{Acknowledgments}

This study was supported by grants from the Broad Medical Research Program; the Italian Ministry of Health; the Fondazione Cariplo; the Italian Association for Cancer Research; the Innovative Medicines Initiative (IMI) Joint Undertaking BTCure (115142); the Fondazione Italiana per la Ricerca Sulle Malattie Apparato Digerente (FIRMAD); and the European Crohn's and Colitis Organisation (ECCO). We thank Ron Haskell (ViraQuest Inc.) for his expert technical assistance with adenovirus preparation. Bronislaw Pytowski (ImClone Systems Inc.) provided the VEGFR3 blocking Ab mF431C1.

Address correspondence to: Silvio Danese, IBD Center, Department of Gastroenterology, Humanitas Clinical and Research Center, Via Manzoni 56, 20089 Rozzano, Milan, Italy. Phone: 390282244771; E-mail: sdanese@hotmail.com.
1. Danese S, Sans M, Fiocchi C. Inflammatory bowel disease: the role of environmental factors. Autoimmun Rev. 2004;3(5):394-400.

2. Molodecky NA, et al. Increasing incidence and prevalence of the inflammatory bowel diseases with time, based on systematic review. Gastroenterology. 2012;142(1):46-54.

3. Podolsky DK. Inflammatory bowel disease. N Engl J Med. 2002;347(6):417-429.

4. Danese S. Nonimmune cells in inflammatory bowel disease: from victim to villain. Trends Immunol. 2008;29(11):555-564.

5. Danese S. Immune and nonimmune components orchestrate the pathogenesis of inflammatory bowel disease. Am J Physiol Gastrointest Liver Physiol. 2011;300(5):G716-G722.

6. Danese S, Fiocchi C. Etiopathogenesis of inflammatory bowel diseases. World J Gastroenterol. 2006;12(30):4807-4812.

7. Danese S, et al. Angiogenesis as a novel component of inflammatory bowel disease pathogenesis. Gastroenterology. 2006;130(7):2060-2073.

8. Danese S, et al. Angiogenesis blockade as a new therapeutic approach to experimental colitis. Gut. 2007;56(6):855-862.

9. Geleff S, Schoppmann SF, Oberhuber G. Increase in podoplanin-expressing intestinal lymphatic vessels in inflammatory bowel disease. Virchows Arch. 2003;442(3):231-237.

10. Pedica F, Ligorio C, Tonelli P, Bartolini S, Baccarini P. Lymphangiogenesis in Crohn's disease: an immunohistochemical study using monoclonal antibody D2-40. Virchows Arch. 2008;452(1):57-63.

11. Chidlow JH, Chidlow JH Jr, Shukla D, Grisham
MB, Kevil CG. Pathogenic angiogenesis in IBD and experimental colitis: new ideas and therapeutic avenues. Am J Physiol Gastrointest Liver Physiol. 2007;293(1):G5-G18.

12. Ganta VC, et al. Angiopoietin-2 in experimental colitis. Inflamm Bowel Dis. 2010;16(6):1029-1039.

13. Jurisic G, Sundberg JP, Detmar M. Blockade of VEGF receptor-3 aggravates inflammatory bowel disease and lymphatic vessel enlargement. Inflamm Bowel Dis. 2012;19(9):1983-1989.

14. Jang JY, et al. Conditional ablation of LYVE-1 ${ }^{+}$ cells unveils defensive roles of lymphatic vessels in intestine and lymph nodes. Blood. 2013;122(13):2151-2161.

15. Heatley RV, Bolton PM, Hughes LE, Owen EW. Mesenteric lymphatic obstruction in Crohn's disease. Digestion. 1980;20(5):307-313.

16. Van Kruiningen HJ, Hayes AW, Colombel JF. Granulomas obstruct lymphatics in all layers of the intestine in Crohn's disease [published online ahead of print April 14, 2014]. APMIS. doi:10.1111/apm.12268.

17. Tonelli F, Giudici F, Liscia G. Is lymphatic status related to regression of inflammation in Crohn's disease? World J Gastrointest Surg. 2012;4(10):228-233.

18. Rahier JF, et al. Decreased lymphatic vessel density is associated with postoperative endoscopic recurrence in Crohn's disease. Inflamm Bowel Dis. 2013;19(10):2084-2090.

19. Wu TF, Carati CJ, Macnaughton WK, von der Weid PY. Contractile activity of lymphatic vessels is altered in the TNBS model of guinea pig ileitis. Am J Physiol Gastrointest Liver Physiol.
2006;291(4):G566-G574

20. Arranz A, et al. Akt1 and Akt2 protein kinases differentially contribute to macrophage polarization. Proc Natl Acad Sci U S A. 2012;109(24):9517-9522.

21. Watanabe N, et al. Elimination of local macrophages in intestine prevents chronic colitis in interleukin-10-deficient mice. Dig Dis Sci. 2003;48(2):408-414

22. Heinsbroek SE, Gordon S. The role of macrophages in inflammatory bowel diseases. Expert Rev Mol Med. 2009;11:e14.

23. Hunter MM, et al. In vitro-derived alternatively activated macrophages reduce colonic inflammation in mice. Gastroenterology. 2010;138(4):1395-1405.

24. Weisser SB, Brugger HK, Voglmaier NS, McLarren KW, van Rooijen N, Sly LM. SHIP-deficient, alternatively activated macrophages protect mice during DSS-induced colitis. J Leukoc Biol. 2011;90(3):483-492.

25. Lawrence T, Gilroy DW. Chronic inflammation: a failure of resolution? Int J Exp Pathol. 2007;88(2):85-94.

26. Jussila L, Alitalo K. Vascular growth factors and lymphangiogenesis. Physiol Rev. 2002;82(3):673-700.

27. Pytowski B, et al. Complete and specific inhibition of adult lymphatic regeneration by a nove VEGFR-3 neutralizing antibody. J Natl Cancer Inst. 2005;97(1):14-21.

28. Kaipainen A, et al. Expression of the fms-like tyrosine kinase 4 gene becomes restricted to lymphatic endothelium during development. Proc Natl Acad Sci U S A. 1995;92(8):3566-3570. 
29. Baluk P, et al. Pathogenesis of persistent lymphatic vessel hyperplasia in chronic airway inflammation. J Clin Invest. 2005;115(2):247-257.

30. Rutkowski JM, Moya M, Johannes J, Goldman $\mathrm{J}$, Swartz MA. Secondary lymphedema in the mouse tail: Lymphatic hyperplasia, VEGF-C upregulation, and the protective role of MMP-9. Microvasc Res. 2006;72(3):161-171.

31. Uzarski J, et al. The resolution of lymphedema by interstitial flow in the mouse tail skin. Am JPhysiol Heart Circ Physiol. 2008;294(3):H1326-H1334.

32. Linares PM, Gisbert JP. Role of growth factors in the development of lymphangiogenesis driven by inflammatory bowel disease: a review. Inflamm Bowel Dis. 2011;17(8):1814-1821.

33. Schoppmann SF, et al. Tumor-associated macrophages express lymphatic endothelial growth factors and are related to peritumoral lymphangiogenesis. Am J Pathol. 2002;161(3):947-956.

34. Skobe M, et al. Concurrent induction of lymphangiogenesis, angiogenesis, and macrophage recruitment by vascular endothelial growth factor-C in melanoma. Am J Pathol. 2001;159(3):893-903.

35. Rahier JF, et al. Increased lymphatic vessel density and lymphangiogenesis in inflammatory bowel disease. Aliment Pharmacol Ther. 2011;34(5):533-543.

36. Tammela T, et al. Angiopoietin-1 promotes lymphatic sprouting and hyperplasia. Blood. 2005;105(12):4642-4648.

37. Rutkowski JM, Boardman KC, Swartz MA. Characterization of lymphangiogenesis in a model of adult skin regeneration. Am J Physiol Heart Circ Physiol. 2006;291(3):H1402-H1410.

38. Alam A, et al. SAR131675, a potent and selective VEGFR-3-TK inhibitor with antilymphangiogenic, antitumoral, and antimetastatic activities. Mol Cancer Ther. 2012;11(8):1637-1649.

39. Lohela M, Saaristo A, Veikkola T, Alitalo K. Lymphangiogenic growth factors, receptors and therapies. Thromb Haemost. 2003;90(2):167-184.

40. Kovi J, Duong HD, Hoang CT. Ultrastructure of intestinal lymphatics in Crohn's disease. $A m J$ Clin Pathol. 1981;76(4):385-394.

41. Robb-Smith AH. A bird's-eye view of Crohn's disease. Proc R Soc Med.1971;64(2):157-161.

42. Van Kruiningen HJ, Colombel JF. The forgotten role of lymphangitis in Crohn's disease. Gut. 2008;57(1):1-4.

43. Liao S, Ruddle NH. Synchrony of high endothelial venules and lymphatic vessels revealed by immunization. J Immunol. 2006;177(5):3369-3379.

44. Sewell GW, Marks DJ, Segal AW. The immunopathogenesis of Crohn's disease: a three-stage model. Curr Opin Immunol. 2009;21(5):506-513.

45. Shih DQ, Targan SR. Immunopathogenesis of inflammatory bowel disease. World J Gastroenterol. 2008;14(3):390-400.

46. Hale LP, Greer PK. A novel murine model of inflammatory bowel disease and inflammation-associated colon cancer with ulcerative colitis-like features. PLoS One. 2012;7(7):e41797.

47. Qualls JE, Kaplan AM, van Rooijen N, Cohen DA. Suppression of experimental colitis by intestinal mononuclear phagocytes. J Leukoc Biol. 2006;80(4):802-815.

48. Bystrom J, et al. Resolution-phase macrophages possess a unique inflammatory phenotype that is controlled by cAMP. Blood. 2008;112(10):4117-4127.

49. Martinez FO, Helming L, Gordon S. Alternative activation of macrophages: an immunologic functional perspective. Annu Rev Immunol. 2009;27:451-483.

50. Takeda K, Kamanaka M, Tanaka T, Kishimoto T, Akira S. Impaired IL-13-mediated functions of macrophages in STAT6-deficient mice. J Immunol.1996;157(8):3220-3222.

51 . Beasley NJ, et al. Intratumoral lymphangiogenesis and lymph node metastasis in head and neck cancer. Cancer Res. 2002;62(5):1315-1320.

52. Padera TP, et al. Lymphatic metastasis in the absence of functional intratumor lymphatics. Science. 2002;296(5574):1883-1886.

53. Tammela $\mathrm{T}$, et al. Therapeutic differentiation and maturation of lymphatic vessels after lymph node dissection and transplantation. Nat Med. 2007;13(12):1458-1466.

54. Spencer DM, Veldman GM, Banerjee S, Willis J, Levine AD. Distinct inflammatory mechanisms mediate early versus late colitis in mice. Gastroenterology. 2002;122(1):94-105.

55. Vowinkel T, Kalogeris TJ, Mori M, Krieglstein CF, Granger DN. Impact of dextran sulfate sodium load on the severity of inflammation in experimental colitis. Dig Dis Sci. 2004;49(4):556-564.

56. Kataru RP, et al. Critical role of CD11b+ macrophages and VEGF in inflammatory lymphangiogenesis, antigen clearance, and inflammation resolution. Blood. 2009;113(22):5650-5659.

57. Makinen T, et al. Inhibition of lymphangiogenesis with resulting lymphedema in transgenic mice expressing soluble VEGF receptor-3. Nat Med. 2001;7(2):199-205.

58. Hoshida T, et al. Imaging steps of lymphatic metastasis reveals that vascular endothelial growth factor-C increases metastasis by increasing delivery of cancer cells to lymph nodes: therapeutic implications. Cancer Res. 2006;66(16):8065-8075.

59. Karkkainen MJ, et al. A model for gene therapy of human hereditary lymphedema. Proc Natl Acad Sci U S A. 2001;98(22):12677-12682.

60. Szuba A, et al. Therapeutic lymphangiogenesis with human recombinant VEGF-C. FASEB J. 2002;16(14):1985-1987.

61. Yoon YS, et al. VEGF-C gene therapy augments postnatal lymphangiogenesis and ameliorates secondary lymphedema. JClin Invest. 2003;111(5):717-725.

62. Huggenberger R, et al. An important role of lymphatic vessel activation in limiting acute inflammation. Blood. 2011;117(17):4667-4678.

63. Jeltsch M, et al. Hyperplasia of lymphatic vessels in VEGF-C transgenic mice. Science. 1997;276(5317):1423-1425.

64. Banks C, Bateman A, Payne R, Johnson P, Sheron N. Chemokine expression in IBD. Mucosal chemokine expression is unselectively increased in both ulcerative colitis and Crohn's disease. JPathol. 2003;199(1):28-35.

65. Garside P. Cytokines in experimental colitis. Clin Exp Immunol. 1999;118(3):337-339.

66. Rogler G, Andus T. Cytokines in inflammatory bowel disease. World J Surg. 1998;22(4):382-389.

67. Gordon S, Martinez FO. Alternative activation of macrophages: mechanism and functions. Immu nity. 2010;32(5):593-604.

68. Stables MJ, et al. Transcriptomic analyses of murine resolution-phase macrophages. Blood. 2011;118(26):e192-e208.

69. Leung G, Wang A, Fernando M, Phan VC, McKay DM. Bone marrow-derived alternatively activated macrophages reduce colitis without promoting fibrosis: participation of IL-10. Am J Physiol Gastrointest Liver Physiol. 2013;304(9):G781-G792.

70. de Jong DJ, et al. No evidence for involvement of IL-4R and CD11B from the IBD1 region and STAT6 in the IBD2 region in Crohn's disease. Eur JHum Genet. 2003;11(11):884-887.

71. Klein $\mathrm{W}$, et al. Interleukin- 4 and interleukin- 4 receptor gene polymorphisms in inflammatory bowel diseases. Genes Immun. 2001;2(5):287-289.

72. Xia B, Crusius JB, Wu J, Zwiers A, van Bodegraven AA, Pena AS. Signal transducer and activator of transcription 6 gene G2964A polymorphism and inflammatory bowel disease. Clin Exp Immunol. 2003;131(3):446-450.

73. Elrod JW, et al. DSS-induced colitis is exacerbated in STAT- 6 knockout mice. Inflamm Bowel Dis. 2005;11(10):883-889.

74. Angeli V, et al. B cell-driven lymphangiogenesis in inflamed lymph nodes enhances dendritic cell mobilization. Immunity. 2006;24(2):203-215.

75. Halin C, Tobler NE, Vigl B, Brown LF, Detmar M. VEGF-A produced by chronically inflamed tissue induces lymphangiogenesis in draining lymph nodes. Blood. 2007;110(9):3158-3167.

76. Berg DJ, et al. Enterocolitis and colon cancer in interleukin-10-deficient mice are associated with aberrant cytokine production and CD4(+) TH1-like responses. JClin Invest. 1996;98(4):1010-1020.

77. Kuhn R, Lohler J, Rennick D, Rajewsky K, Muller W. Interleukin-10-deficient mice develop chronic enterocolitis. Cell. 1993;75(2):263-274.

78. Wirtz S, Neufert C, Weigmann B, Neurath MF. Chemically induced mouse models of intestinal inflammation. Nat Protoc. 2007;2(3):541-546.

79. Cooper HS, Murthy SN, Shah RS, Sedergran DJ. Clinicopathologic study of dextran sulfate sodium experimental murine colitis. Lab Invest. 1993;69(2):238-249.

80. Scaldaferri F, et al. Crucial role of the protein C pathway in governing microvascular inflammation in inflammatory bowel disease. JClin Invest. 2007;117(7):1951-1960.

81. Schacht V, Dadras SS, Johnson LA, Jackson DG, Hong YK, Detmar M. Up-regulation of the lymphatic marker podoplanin, a mucin-type transmembrane glycoprotein, in human squamous cell carcinomas and germ cell tumors. Am J Pathol. 2005;166(3):913-921. 